\title{
Numerical simulation of non-linear elastic flows with a general collocated finite-volume method
}

\author{
P.J. Oliveira ${ }^{\mathrm{a}, *}$, F.T. Pinho ${ }^{\mathrm{b}}$, G.A. Pinto ${ }^{\mathrm{c}}$ \\ a Departamento de Engenharia Electromecânica, Universidade da Beira Interior Rua Marquês D’Ávila e Bolama, \\ 6200 Covilhã, Portugal \\ ${ }^{\mathrm{b}}$ Departamento de Engenharia Mecânica e Gestão Industrial Faculdade de Engenharia da Universidade do Porto, \\ Rua dos Bragas, 4099 Porto Codex, Portugal \\ c Departamento de Engenharia Química, Instituto Superior de Engenharia do Porto, Rua de São Tomé, \\ 4200 Porto Codex, Portugal
}

Received 13 June 1997; received in revised form 14 January 1998

\begin{abstract}
This paper reports the development and application of a finite-volume based methodology for the calculation of the flow of fluids which follow differential viscoelastic constitutive models. The novelty of the method lies on the use of the non-staggered grid arrangement, in which all dependent variables are located at the center of the control volumes, thus greatly simplifying the adoption of general curvilinear coordinates. The pressure-velocity-stress decoupling was removed by the development of a new interpolation technique inspired on that of Rhie and Chow, AIAA 82 (1982) 998. The differencing schemes are second order accurate and the resulting algebraic equations for each variable are solved in a segregated way (decoupled scheme). The numerical formulation especially designed for the interpolation of the stress field was found to work well and is shown to be indispensable for accurate results. Calculations have been carried out for two problems: the entry flow problem of Eggleton et al., J. Non-Newtonian Fluid Mech. 64 (1996) 269, with orthogonal and non-orthogonal meshes; and the bounded and unbounded flows around a circular cylinder. The results of the simulations compare favourably with those in the literature and iterative convergence has been attained for Deborah and Reynolds numbers similar to, or higher than, those reported for identical flow problems using other numerical methods. The application of the method with non-orthogonal coordinates is demonstrated. The entry flow problem is studied in more detail and for this case differences between Newtonian and viscoelastic fluids are identified and discussed. Viscoelasticity is shown to be responsible for the development of very intense normal stresses, which are tensile in the wall region. As a consequence, the viscoelastic fluid is more intensely decelerated in the wall region than the Newtonian fluid, thus reducing locally the shear rates and the role of viscosity in redeveloping the flow. A layer of high stress-gradients is formed at the wall leading edge and is convected below and away from the wall; its effect is to intensify the aforementioned deviation of elastic fluid from the wall. (C) 1998 Elsevier Science B.V. All rights reserved.
\end{abstract}

\footnotetext{
* Corresponding author.
}

0377-0257/98/\$19.00 (C) 1998 Elsevier Science B.V. All rights reserved.

PII S0377-0257(98)00082-2 
Keywords: Finite-volume; Collocated grids; Non-orthogonal coordinates; Upper convected Maxwell model; Slip-stick flow; Decoupled method

\section{Introduction}

Finite-volume methods for the calculation of fluid flow can be advantageous in terms of computer space and time requirements, as well as in terms of numerical stability, when compared to finite-element methods [3]. Various such finite-volume methods have been developed and used in Newtonian fluid mechanics from the late sixties on, as can be assessed by a wealth of literature on the subject $[4,5]$.

In spite of its many advantages, finite-volume methods have seen a limited application to the computation of Non-Newtonian viscoelastic fluid flows, and it is only recently that various research groups have started using this class of numerical methods. This state of things has a probable origin in that a large number of researchers working in the field of computational rheology tended to have a background on solid mechanics, rather than on classical computational fluid dynamics where finite-volume methods are often used.

One of the first recent numerical works based on the finite-volume approach was the investigation of the flow of an upper convected Maxwell (UCM) model fluid around a circular cylinder [6]. A staggered orthogonal grid and the semi-implicit method for pressure linked equations revised (SIMPLER) algorithm was used and adapted for the calculation of the stress components. The inertia terms in the momentum equation were neglected as the calculations were limited to low Reynolds number flows with convergence attained for Weissenberg numbers up to 10 .

The inclusion of inertia terms in the momentum equation is indispensable for the computation of higher Reynolds number flows, however, this may have adverse consequences on the iterative behaviour of methods designed for creeping flow conditions. Therefore, it is important to consider inertia from the outset when developing general algorithms, as carried by Yoo and $\mathrm{Na}$ in their predictions of the sudden contraction flow [7]. However, like $\mathrm{Hu}$ and Joseph [6], Yoo and $\mathrm{Na}$ have only considered first order interpolation schemes, which are known to cause severe numerical diffusion whenever the flow is not aligned with the grid orientation [8]. The use of staggered grids has also been the normal practice in the above works, as well as in other calculations of Non-Newtonian flows with finite-volume methods [9-11]. All of these groups relied upon various forms of the well-known pressure correction strategy applied in staggered grids [4].

Relevant industrial geometries are usually not so simple as to allow the direct use of the above techniques, thus a more general formulation based on collocated non-orthogonal grids is required for handling the flow of Non-Newtonian fluids in complex geometries. This was recently recognized by Huang et al. [3], who used a non-structured method for predicting the flow of Phan-Thien-Tanner fluids in eccentric bearings. Besides neglecting the inertia terms in the momentum equation, their formulation lacks some of the elegance and generality of the approach now followed in most classical fluid dynamics studies, heavily rooted in the collocated grid arrangement [5]. 
Non-Newtonian viscoelastic fluids have a complex rheological behaviour, which requires the adoption of non-linear constitutive equations for realistic predictions. The use of simple models, such as the UCM or White-Metzner models, can only partially predict real fluid rheology. Nevertheless, these models are very challenging from the numerical point of view, due to their possession of the inherent difficulties associated with stress singular behaviour near sharp edges or with sudden changes in boundary conditions. Thus, they are very suitable in the development of accurate, robust numerical methods that can be upgraded later to more complex and realistic constitutive equations. Indeed, it is generally accepted [3] that the UCM equations pose the most severe numerical difficulties compared with other constitutive differential models, and it is safe to say that a good numerical method developed for UCM fluids is expected to work well with other models.

The objective of this paper is to present in detail a finite-volume based numerical method for non-orthogonal collocated grids, and to include second-order accurate interpolation schemes, for the prediction of Non-Newtonian flows. The basic constitutive model adopted is the UCM, however, both the viscosity and the relaxation time can be prescribed functions of the rate of strain, as in the White-Metzner model.

As test cases, two problems have been considered: the entry flow in a two dimensional planar channel, with both orthogonal and a non-orthogonal grids, and the flow around a cylinder in a channel with area blockages of 30 and $50 \%$, as well as the unbounded flow case.

In Section 2, the equations are presented and their discretization outlined. Subsequently, the numerical method, the interpolation schemes, the methodology developed to address the pressure-velocity-stress coupling and the definition of boundary conditions are presented and discussed in some detail. The numerical procedure is then applied to investigate numerically the two-dimensional Poiseuille entry flow and the flow around a cylinder for the UCM fluid.

\section{Governing equations}

The basic equations are those for three-dimensional, incompressible and isothermal laminar flow of a UCM fluid. Unless otherwise stated, the summation convention for repeated indices

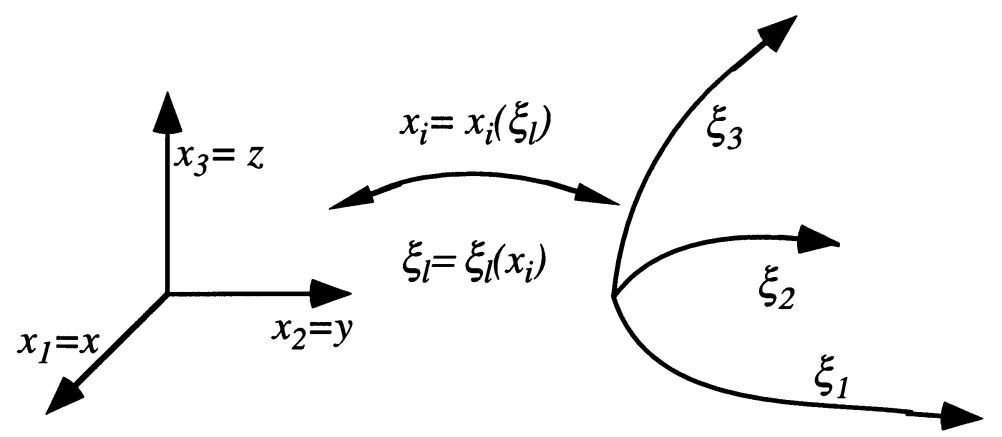

Fig. 1. Schematic representation of the transformation of a rectangular Cartesian coordinate system to a non-orthogonal coordinate system which follows the mesh lines. 
will apply to either Cartesian $(i, j, \ldots)$ or non-Cartesian $(l, m, \ldots)$ directions Fig. 1. The continuity equation is

$$
\frac{\partial u_{i}}{\partial x_{i}}=0
$$

and the momentum conservation equation is

$$
\frac{\partial \rho u_{i}}{\partial t}+\frac{\partial \rho u_{j} u_{i}}{\partial x_{j}}=-\frac{\partial p}{\partial x_{i}}+\frac{\partial \tau_{i j}}{\partial x_{j}}+\rho g_{i}
$$

where the extra stress tensor $\tau_{i j}$ is defined by the UCM constitutive equation:

$$
\tau_{i j}+\lambda \tau_{(1) i j}=\eta\left[\frac{\partial u_{i}}{\partial x_{j}}+\frac{\partial u_{j}}{\partial x_{i}}\right]-\frac{2}{3} \eta \frac{\partial u_{k}}{\partial x_{k}} \delta_{i j}
$$

In the constitutive equation, $\lambda$ is the relaxation time, $\eta$ the shear viscosity and the last term on the right hand side of Eq. (3) is zero for incompressible fluids, like those analysed here. However, it is nevertheless retained as it is not exactly zero in the numerical approximation and it improves the convergence rate. In (3), $\tau_{(1) i j}$ denotes Oldroyd's upper convected derivative of $\tau_{i j}$ given by

$$
\tau_{(1) i j}=\frac{\partial \tau_{i j}}{\partial t}+u_{k} \frac{\partial \tau_{i j}}{\partial x_{k}}-\tau_{j k} \frac{\partial u_{i}}{\partial x_{k}}-\tau_{i k} \frac{\partial u_{j}}{\partial x_{k}} .
$$

The mass and momentum conservation equations and the constitutive equation obey the principle of invariance and are written for an orthogonal coordinate system $\left(x_{1}, x_{2}, x_{3}\right)$. Their discretization on a general computational finite-volume mesh composed of non-orthogonal six-faced cells requires that they be transformed first to a general non-orthogonal coordinate system $\left(\xi_{1}, \xi_{2}, \xi_{3}\right)$ and it is advantageous from a numerical point of view to write the equations in a strong conservation form, which helps ensuring that the final algebraic equations will retain conservatiness. The required transformation rules are [12]

$$
\begin{aligned}
& \frac{\partial}{\partial t}=\frac{1}{J} \frac{\partial}{\partial t} J, \\
& \frac{\partial}{\partial x_{i}}=\frac{\partial}{\partial \xi_{l}} \frac{\partial \xi_{l}}{\partial x_{i}}=\frac{1}{J} \frac{\partial}{\partial \xi_{l}} \beta_{l i},
\end{aligned}
$$

where $J$ is the Jacobian of the transformation $x_{i}=x_{i}\left(\xi_{l}\right)$ and $\beta_{l i}$ are metric coefficients defined as the cofactor of $\partial x_{i} / \partial \xi_{l}$ and readily interpreted as area components after integration. After applying the rules of transformation (7) to Eqs. (1)-(4), these can be written in compact form, as follows:

- continuity

$$
\frac{\partial}{\partial \xi_{l}}\left(\rho \beta_{l j} u_{j}\right)=0
$$

- momentum 


$$
\frac{\partial}{\partial t}\left(J \rho u_{i}\right)+\frac{\partial}{\partial \xi_{l}}\left(\rho \beta_{l j} u_{j} u_{i}\right)=-\beta_{l i} \frac{\partial p}{\partial \xi_{l}}+\frac{\partial}{\partial \xi_{l}}\left(\beta_{l j} \tau_{i j}\right)+J \rho g_{i}
$$

- constitutive equation

$$
\begin{aligned}
& J \tau_{i j}+\lambda \frac{\partial}{\partial t}\left(J \tau_{i j}\right)+\lambda \frac{\partial}{\partial \xi_{l}}\left(\beta_{l k} u_{k} \tau_{i j}\right) \\
& =\eta\left[\beta_{l j} \frac{\partial u_{i}}{\partial \xi_{l}}+\beta_{l i} \frac{\partial u_{j}}{\partial \xi_{l}}\right]+\lambda\left[\left(\beta_{l k} \tau_{k j}\right) \frac{\partial u_{i}}{\partial \xi_{l}}+\left(\beta_{l k} \tau_{k i}\right) \frac{\partial u_{j}}{\partial \xi_{l}}\right]-\frac{2}{3} \eta \beta_{l k} \frac{\partial u_{k}}{\partial \xi_{l}} \delta_{i j} .
\end{aligned}
$$

More details on this can be found in [13]. In Eq. (9), the terms which will be dealt with implicitly in the numerical procedure are written on the left hand side. It is well known that diffusion is essential to promote the stability of finite-volume schemes when applied to transport equations, and since viscous diffusion is not explicitly present in Eq. (9), it was decided to add an ordinary diffusion term to both sides of the momentum equation. This procedure departs from the usual method in Non-Newtonian calculations, where the stress term is split into viscous and elastic contributions and the viscous contribution is then made to appear explicitly in the momentum equation. The two procedures may lead to analogous equations in some instances. However. it should be noted that the inclusion of the normal viscous term is just for numerical convenience here, based on experience gained in Newtonian flow calculations with the deferred correction approach [14]. Eq. (9) is thus replaced by

$$
\begin{aligned}
& \frac{\partial}{\partial t}\left(J \rho u_{i}\right)+\frac{\partial}{\partial \xi_{l}}\left(\rho \beta_{l j} u_{j} u_{i}\right)-\frac{\partial}{\partial \xi_{l}}\left(\frac{\eta}{J} \beta_{\mathbf{l} j} \beta_{\mathbf{l} j} \frac{\partial u_{i}}{\partial \xi_{l}}\right)=-\beta_{l i} \frac{\partial p}{\partial \xi_{l}}+\frac{\partial}{\partial \xi_{l}}\left(\beta_{l j} \tau_{i j}\right)+J \rho g_{i}- \\
& \frac{\partial}{\partial \xi_{l}}\left(\frac{\eta}{J} \beta_{\mathbf{l} j} \beta_{\mathbf{l} j} \frac{\partial u_{i}}{\partial \xi_{l}}\right) \quad(\mathbf{l}=l \text { and no summation over subscript } \mathbf{l}),
\end{aligned}
$$

in which all terms on the left hand side of the equality sign are implicitly dealt with (incorporated into the coefficients of the algebraic equations) and those on the right hand side explicitly (incorporated into the source term of the algebraic equation), as explained hereafter.

\section{Numerical method}

\subsection{Introduction and notation}

In the finite-volume method, the computational domain is divided into contiguous 6-faced cells and the differential equations are integrated over each cell so that full conservativeness is ensured. This is accomplished by applying Gauss' theorem, as explained in [4], with the necessary modifications due to the use of the collocated mesh arrangement in which all variables are stored at the centre-of-cell location. In general, all terms are discretised by means of central differences, which amounts to using linear interpolation to evaluate variable values in locations where they are not stored, except for the convection terms which are approximated by the linear-upwind differencing scheme (LUDS). This is a generalisation of the well-known upwind differencing scheme (UDS), where the value of a convected variable at a cell face location is given by its value at the first upstream cell centre. In the LUDS scheme, the value of that 

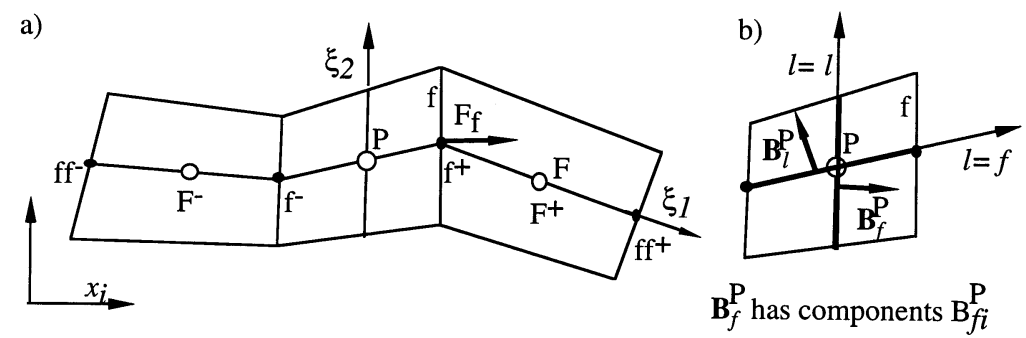

Fig. 2. Some nomenclature: (a) general and neighbouring cells; (b) area vectors and components.

convected variable at the same cell face is given by a linear extrapolation based on the values of the variable at the two upstream cells. It is, in general, second-order accurate, as compared with first-order accuracy of UDS, and thus, its use reduces the problem of numerical diffusion. Note that, in the present formulation, no use is made of any explicit artificial-diffusion added term as is often the case in finite-element methods. The end result of the discretisation is a set of algebraic equations relating centre-of-cell values of the unknown variables to their values at near-by cells, to be solved by an existing conjugate-gradient method for solving linear sets of equations.

The integration of the governing equations written in generalised co-ordinates, Eqs. (8), (10) and (11), is straightforward after an acquaintance with the nomenclature introduced in Fig. 2. In fact, it suffices to replace: the $\beta_{l i}$ coefficients by area components of the surface along direction $l$, denoted $B_{l i}$; the Jacobian $J$ by the cell volume $\mathscr{V}$; and the derivatives $\partial / \partial \xi_{l}$ by differences between values along direction $l$, thus

$$
\frac{\partial \Phi}{\partial \xi_{l}}=[\Delta \Phi]_{l} \equiv \Phi_{l^{+}}-\Phi_{l^{-}} .
$$

Both differences, and the area components, can be evaluated at a cell-centre location (superscript P),

$$
[\Delta \Phi]_{f}^{\mathrm{P}} \equiv \Phi_{\mathrm{f}^{+}}-\Phi_{\mathrm{f}^{-}} \quad \text { and } \quad B_{f i}^{\mathrm{P}}
$$

or at a cell face (superscript $f$ ),

$$
[\Delta \Phi]_{f}^{\mathrm{f}} \equiv \Phi_{\mathrm{F}}-\Phi_{\mathrm{P}} \quad \text { and } \quad B_{f i}^{\mathrm{f}} \text {. }
$$

This is used here to avoid too many subscripts which may become confusing; otherwise, location is indicated with a subscript. ' $\mathrm{F}$ ' denotes the first cell neighbour to the general cell ' $\mathrm{P}$ ' along direction of face ' $f$ ' (Fig. 2); double characters ('FF' and 'ff') are used to indicate the second neighbour along the same direction ' $f$ ', and its use is restricted to the LUDS scheme for the convection terms. In the discretised equations, variables at a general cell ' $\mathrm{P}$ ' and at its six neighbours $(\mathrm{F}=1-6$, for $\mathrm{W}, \mathrm{E}, \mathrm{S}, \mathrm{N}, \mathrm{B}$ and $\mathrm{T}$ with compass notation: west, east, south, north, bottom and top, i.e. for $l= \pm 1,2$ and 3, respectively) are treated implicitly and form the main stencil in the discretisation. The six far-away neighbours (FF $=1-6$, for $\mathrm{WW}, \mathrm{EE}, \mathrm{SS}, \mathrm{NN}, \mathrm{BB}$ and TT), used in the LUDS scheme, give rise to contributions which are incorporated into the so-called source term and are treated explicitly. Thus, the linearized sets of equations for each 
dependent variable, which need to be solved at every time step, have a well-structured matrix with 7 non-zero diagonals - this is one of the main differences with the finite-element method which gives rise to banded matrices with no particular structure inside the band.

After this brief introduction, details of the discretisation are now given for each equation.

\subsection{Discretization of the equations}

\subsubsection{Continuity equation}

The continuity equation (1) is volume integrated and discretised as follows (sums are explicitly indicated in the discretized equations):

$$
\int_{\mathscr{V}_{\mathrm{P}}} \frac{\partial}{\partial \xi_{l}}\left(\rho \beta_{l j} u_{j}\right) \mathrm{d} \mathscr{V}=\sum_{l=1}^{3}\left[\Delta\left(\sum_{j} \rho B_{l j} u_{j}\right)\right]_{l}^{\mathrm{P}}=\sum_{\mathrm{f}=1}^{6}\left(\sum_{j} \rho B_{f j}^{\mathrm{f}} \tilde{u}_{j, \mathrm{f}}\right) .
$$

Here, the sum of differences centred at cell centre $\mathrm{P}$ has been transformed into a sum of contributions arising from the six cell faces, f. The notation $\tilde{u}_{j, \mathrm{f}}$ for the cell face velocity component $u_{j}$ means that this cannot be computed from simple linear interpolation, in which case no special symbol would be required according to our nomenclature, but need to be evaluated via a Rhie and Chow [1] interpolation, to be explained below in Section 3.4. Mass flow rates through a cell face are thus defined as

$$
F_{\mathrm{f}}=\sum_{j} \rho B_{f j}^{\mathrm{f}} \tilde{u}_{j, \mathrm{f}}
$$

and the discretised continuity Eq. (15) can be rewritten as

$$
\sum_{\mathrm{f}=1}^{6} F_{\mathrm{f}}=0
$$

expressing the fact that the sum of in-coming mass flow rates equals the sum of out-going flow rates.

\subsubsection{Momentum equation}

The integration of each term in Eq. (11), starting from left to right, gives the results below.

- Inertia term

$$
\int_{\mathscr{V}_{\mathrm{P}}} \frac{\partial}{\partial t}\left(J \rho u_{i}\right) \mathrm{d} \mathscr{V}=\frac{\rho \mathscr{V}_{\mathrm{P}}}{\delta t}\left(u_{i, \mathrm{P}}-u_{i, \mathrm{P}}^{\mathrm{o}}\right),
$$

where $u_{i, \mathrm{P}}^{\mathrm{o}}$ is the velocity at cell $\mathrm{P}$ at the previous time level. The present method is fully implicit meaning that all variables without a time-level superscript are assumed to pertain to the new time-level.

- Convection term

As in Eq. (15), we have

$$
\int_{\mathscr{V}_{\mathrm{P}}} \frac{\partial}{\partial \xi_{l}}\left(\rho \beta_{l j} u_{j} u_{i}\right) \mathrm{d} \mathscr{V}=\sum_{l=1}^{3}\left[\Delta\left(\sum_{j}\left(\rho B_{l j} u_{j}\right) u_{i}\right)\right]_{l}^{\mathrm{P}}=\sum_{\mathrm{f}=1}^{6} F_{\mathrm{f}} \hat{u}_{i, \mathrm{f}}
$$


with the cell face mass fluxes defined as in Eq. (16) and the convected velocity at face $\mathrm{f}, \hat{u}_{i, \mathrm{f}}$, being given according to the discretisation scheme adopted for the convective terms. For the upwind differencing scheme, $\hat{u}_{i, \mathrm{f}}$ is simply the velocity at the centre of the cell in the upstream direction:

$$
\hat{u}_{i, \mathrm{f}}=u_{i, \mathrm{P}} \quad\left(\text { for } F_{\mathrm{f}}>0\right) \quad \text { and } \quad \hat{u}_{i, \mathrm{f}}=u_{i, \mathrm{~F}} \quad\left(F_{\mathrm{f}}<0\right) \quad \text { (see Fig. 2). }
$$

This can generally be written by expressing the convection fluxes of momentum as

$$
F_{\mathrm{f}} \hat{u}_{i, \mathrm{f}}=F_{\mathrm{f}}^{+} u_{i, \mathrm{P}}+F_{\mathrm{f}}^{-} u_{i, \mathrm{~F}} \quad \text { where } \quad F_{\mathrm{f}}^{+} \equiv \operatorname{Max}\left(F_{\mathrm{f}}, 0\right) \quad \text { and } \quad \mathrm{F}_{\mathrm{f}}^{-} \equiv \operatorname{Min}\left(F_{\mathrm{f}}, 0\right) .
$$

In the case of the LUDS scheme, the expression of the convection flux is more complicated and is left for Section 3.3.

- Ordinary diffusion term added to the left side of the momentum equation

$$
-\int_{\mathscr{V}_{\mathrm{P}}} \frac{\partial}{\partial \xi_{l}}\left(\frac{\eta}{J} \beta_{\mathrm{l} j} \beta_{\mathrm{l} j} \frac{\partial u_{i}}{\partial \xi_{l}}\right) d \mathscr{V}=-\sum_{\mathrm{f}=1}^{6} \frac{\eta_{\mathrm{f}}}{\mathscr{V}_{\mathrm{f}}} B_{\mathrm{f}}^{2}\left[\Delta u_{i}\right]_{f}^{\mathrm{f}}=-\sum_{\mathrm{f}=1}^{6} D_{\mathrm{f}}\left(u_{i, \mathrm{~F}}-u_{i, \mathrm{P}}\right)
$$

where the surface area of the cell face is $B_{\mathrm{f}}=\left(\sum_{j} B_{f j}^{\mathrm{f}} B_{f j}^{\mathrm{f}}\right)^{1 / 2}$, the volume of a pseudo cell centered at the face is $\mathscr{V}_{\mathrm{f}}=\sum_{j=1}^{3} B_{f j}^{\mathrm{f}}\left[\Delta x_{j}\right]_{f}^{\mathrm{f}}$, and $D_{\mathrm{f}} \equiv \eta_{\mathrm{f}} B_{\mathrm{f}}^{2} / \mathscr{V}_{\mathrm{f}}$ is a diffusion conductance.

- Pressure gradient term

$$
\int_{\mathscr{V}_{\mathbf{P}}} \beta_{l i} \frac{\partial p}{\partial \xi_{l}} \mathrm{~d} \mathscr{V}=-\sum_{l=1}^{3} B_{l i}^{\mathrm{P}}[\Delta p]_{l}^{\mathrm{P}} \equiv S_{u_{i}-\text { pressure }}
$$

- Stress-divergence term

$$
\int_{\mathscr{V}_{\mathrm{P}}} \frac{\partial}{\partial \xi_{l}}\left(\beta_{l j} \tau_{i j}\right) \mathrm{d} \mathscr{V}=\sum_{\mathrm{f}=1}^{6} B_{f j}^{\mathrm{f}} \tilde{\tau}_{i j, \mathrm{f}} \equiv S_{u_{i}-\text { stress }}
$$

where, like with the face velocity in the continuity equation beforehand, the cell-face stress requires a special interpolation method due to the use of the collocated mesh arrangement. The way to do this is precisely one of the contributions of the present work and it is essential for the applicability of this method; the solution is given in Section 3.5.

- Gravity or body-force term:

$$
\int_{\mathscr{V}_{\mathrm{P}}} J \rho g_{i} \mathrm{~d} \mathscr{V}=\rho \mathscr{V}_{\mathrm{P}} g_{i} \equiv S_{u_{i}-\text { gravity }}
$$

- Ordinary diffusion term added to the right hand side of the momentum equation. In order to avoid inconsistencies, this term is treated exactly as the diffusion term above (Eq. (22)), yielding

$$
-\sum_{\mathrm{f}=1}^{6} D_{\mathrm{f}}\left(u_{i, \mathrm{~F}}-u_{i, \mathrm{P}}\right) \equiv S_{u_{i}-\text { diffusion }}
$$

The only difference lies on the way the two terms are dealt with: while the former is treated implicitly, with $D_{\mathrm{f}}$ added to the coefficient $a_{\mathrm{F}}$, the latter is added to the source term and lags 
by a time level during the time advancement of the equations. When the steady state solution is attained, the two terms exactly cancel each other.

The final form of the momentum balance is arrived at after re-grouping the various terms, to give

$$
a_{P} u_{i, \mathrm{P}}-\sum_{\mathrm{F}} a_{\mathrm{F}} u_{i, \mathrm{~F}}=S_{u_{i}}+\frac{\rho \mathscr{V}_{\mathrm{P}}}{\delta t} u_{i, \mathrm{P}}^{\mathrm{o}}
$$

where the coefficients $a_{\mathrm{F}}$ consist of convection ( $a_{\mathrm{F}}^{C}$, here based on UDS) and diffusion contributions $\left(a_{\mathrm{F}}^{D}\right)$ :

$$
\begin{aligned}
& a_{\mathrm{F}}=a_{\mathrm{F}}^{D}+a_{\mathrm{F}}^{C} \text {, with } a_{\mathrm{F}}^{D}=D_{\mathrm{f}} \text { and } \quad a_{\mathrm{F}}^{C}=+F_{\mathrm{f}}^{+} \text {(for a negative face, } \mathrm{f}^{-} \text {) } \\
& a_{\mathrm{F}}^{C}=-F_{\mathrm{f}}^{-}\left(\text {for a positive face, } \mathrm{f}^{+}\right. \text {) }
\end{aligned}
$$

The central coefficient is

$$
a_{\mathrm{P}}=\frac{\rho \mathscr{V}_{\mathrm{P}}}{\delta t}+\sum_{\mathrm{F}} a_{\mathrm{F}}
$$

and the total source term is given by the sum

$$
S_{u_{i}}=S_{u_{i}-\text { pressure }}+S_{u_{i}-\text { gravity }}+S_{u_{i}-\text { stress }}+S_{u_{i}-\text { diffusion }}
$$

\subsubsection{Constitutive equation}

The three terms on the left hand side of the equality sign of Eq. (10) are discretised as the gravity term above (25), the inertia term (18) and the convection term (19), respectively, and do not present any additional difficulty. It is only noted that in all terms the velocity component $u_{i}$ is replaced by $\tau_{i j}$ and the mass flow rates in the convective fluxes, defined in Eq. (16), should be multiplied by the ratio of the relaxation time $\lambda$ to the density $\rho$ (compare the convective fluxes in Eqs. (9) and (10)). Following the same approach as above, the source term in the stress constitutive equation becomes

$$
\begin{aligned}
& S_{\tau i j}=\eta_{\mathrm{P}} \sum_{l}^{3}\left(B_{l j}^{\mathrm{P}}\left[\Delta u_{i}\right]_{l}^{\mathrm{P}}+B_{l i}^{\mathrm{P}}\left[\Delta u_{j}\right]_{l}^{\mathrm{P}}\right)+\lambda_{P} \sum_{l}^{3}\left(\left(\sum_{k}^{3} B_{l k}^{\mathrm{P}} \tau_{i k, \mathrm{P}}\right)\left[\Delta u_{j}\right]_{l}^{\mathrm{P}}+\left(\sum_{k}^{3} B_{l k}^{\mathrm{P}} \tau_{j k, \mathrm{P}}\right)\left[\Delta u_{i}\right]_{l}^{\mathrm{P}}\right)- \\
& \frac{2}{3} \eta_{\mathrm{P}}\left(\sum_{l}^{3} \sum_{k}^{3} B_{l k}^{\mathrm{P}}\left[\Delta u_{k}\right]_{l}^{\mathrm{P}}\right) \delta_{i j} .
\end{aligned}
$$

The linearized form of the stress equation is, therefore,

$$
a_{\mathrm{P}}^{\tau} \tau_{i j \mathrm{P}}-\sum_{\mathrm{F}} a_{\mathrm{F}}^{\tau} \tau_{i j, \mathrm{~F}}=S_{\tau_{i j}}+\frac{\lambda_{\mathrm{P}} \mathscr{V}_{P}}{\delta t} \tau_{i j, \mathrm{P}}^{\mathrm{o}}
$$

with the coefficients $a_{\mathrm{F}}^{\tau}$ consisting of the convective coefficients in Eq. (28) multiplied by $\lambda / \rho$, for the reasons just explained, and the central coefficient is

$$
a_{\mathrm{P}}^{\tau}=\mathscr{V}_{\mathrm{P}}+\sum_{\mathrm{F}} a_{\mathrm{F}}^{\tau}+\frac{\lambda_{P} \mathscr{V}_{\mathrm{P}}}{\delta t}
$$


Note that these coefficients are the same for all the six stress components, hence greatly reducing the storage requirements. For a Newtonian fluid $(\lambda=0)$ all coefficients $a_{\mathrm{F}}^{\tau}$ vanish and the stress Eq. (32) reduces to an explicit algebraic relationship giving the usual Newtonian stresses - in this case there is no need to solve (32) as a matrix equation, the solution is obtained in one step without necessity to take any particular precautions.

\subsection{Linear-upwind differencing scheme (LUDS) for convection}

The convection terms in the momentum and stress equations involve first derivatives of the quantities being convected and thus require some form of interpolation in order to determine these quantities at cell faces. In the previous section, the upwind differencing scheme has been used leading to the convected velocities defined by Eq. (20). UDS is the most stable of the existing schemes for convection, but since it is only first order in space it may lead to numerical diffusion. An obvious extension of the UDS is the linear upwind differencing scheme (LUDS) in which two upstream values, instead of just one as in the UDS, are used to determine the face values by linear extrapolation [5]. Referring to the one-dimensional example in Fig. 3, the velocity at the 'east' face is now given by

$$
\hat{u}_{e}=u_{\mathrm{P}}+\left(1-L_{\mathrm{w}}\right)\left(u_{\mathrm{P}}-u_{\mathrm{W}}\right) \quad\left(F_{e}>0\right),
$$

where $L_{\mathrm{w}}$ is a linear interpolation weighting factor defined as $L_{\mathrm{w}}=\frac{l_{W}}{l_{W}+l_{\mathrm{P}}}\left(l_{\mathrm{W}}, l_{\mathrm{P}}\right.$ are cell widths). If the flow was from 'east' to 'west' (the negative $\xi_{1}$ direction), then a similar extrapolation would give

$$
\hat{u}_{e}=u_{\mathrm{E}}+\left(1-L_{e e}\right)\left(u_{\mathrm{E}}-u_{\mathrm{EE}}\right) \quad\left(F_{e}<0\right) .
$$

With this scheme, the convective fluxes at all faces could be constructed to derive the convection coefficients given below.

In the general case, the linear interpolation factors are written as

$$
L_{\mathrm{f}}^{+}=\frac{[\Delta l]_{f}^{\mathrm{P}}}{[\Delta l]_{f}^{\mathrm{P}}+[\Delta l]_{f}^{\mathrm{F}}}, \quad L_{\mathrm{f}}^{-}=1-L_{\mathrm{f}}^{+},
$$

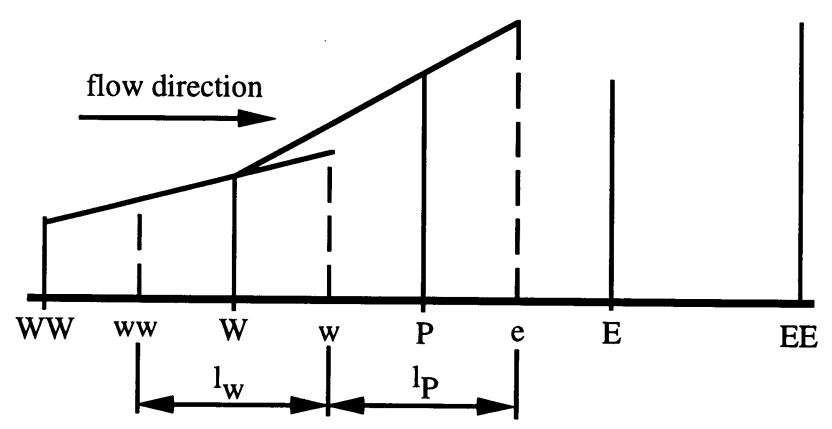

Fig. 3. Schematic representation of the linear upwind scheme. 
where $\Delta l$ is the cell length along direction $f$, so that a quantity $\phi$ to be determined by linear interpolation at the cell face $\mathrm{f}$ is given by $\phi_{\mathrm{f}}=L_{\mathrm{f}}^{+} \phi_{F}+L_{\mathrm{f}}^{-} \phi_{P}$. The new convective coefficients based on LUDS at a positive face $\left(\mathrm{f}^{+}\right)$can be shown to be, for the near-neighbour cells $(\mathrm{f}=\mathrm{W}$, E, S, N, B and T)

$$
a_{f^{+}}^{C}=-\left[L_{\mathrm{ff}+}^{+} F_{\mathrm{f}^{+}}^{-}+L_{\mathrm{f}^{+}}^{+} F_{\mathrm{f}^{-}}^{-}+F_{\mathrm{f}^{+}}^{-}\right],
$$

and for the far-neighbour cells $(\mathrm{f}=\mathrm{WW}, \mathrm{EE}, \mathrm{SS}, \mathrm{NN}, \mathrm{BB}, \mathrm{TT})$

$$
a_{f f^{+}}^{C}=+\left[L_{\mathrm{ff}^{+}}^{+} F_{\mathrm{f}^{+}}^{-}\right],
$$

where $F_{\mathrm{f}}^{+} \equiv \operatorname{Max}\left(F_{\mathrm{f}}, 0\right)$ and $F_{\mathrm{f}}^{-} \equiv \operatorname{Min}\left(F_{\mathrm{f}}, 0\right)$. For a negative face (f ${ }^{-}$in Fig. 2$)$, the same expressions hold with all $+/-$ signs interchanged. The compact notation here utilised is somewhat difficult to interpret, however, it is the only way to write these expressions concisely. With these coefficients, the global convective flux contribution to the $i$-momentum equation (Eq. (19)), becomes

$$
\sum_{\mathrm{f}} F_{\mathrm{f}} \hat{u}_{i, \mathrm{f}}=\sum_{\mathrm{F}} \mathrm{a}_{\mathrm{F}}^{C}\left(u_{i, \mathrm{P}}-u_{i, \mathrm{~F}}\right)+\sum_{\mathrm{FF}} a_{\mathrm{FF}}^{C}\left(u_{i, \mathrm{P}}-u_{i, \mathrm{FF}}\right) .
$$

The first term in the right hand side is treated implicitly, and the $a_{\mathrm{F}}^{C}$ coefficients (now given by Eq. (36a) for a positive face) are added to the diffusion coefficients $a_{\mathrm{F}}^{D}$ to form the usual $a_{\mathrm{F}}$ coefficients, as in Eq. (28). The second term in Eq. (37) is shifted to the right hand side of the momentum equation, with $a_{\mathrm{FF}}^{C}$ given by Eq. (36b) for a positive face, and is treated explicitly through the standard linearization procedure [4]:

$$
\sum_{\mathrm{FF}} a_{\mathrm{FF}}^{C}\left(u_{i, \mathrm{P}}-u_{i, \mathrm{FF}}\right)=S_{u_{i-\mathrm{LUDS}}}-S_{\mathrm{P}} u_{i, \mathrm{P}} \equiv\left(\sum_{\mathrm{FF}} a_{\mathrm{FF}}^{C} u_{i, \mathrm{FF}}\right)-\left(\sum_{\mathrm{FF}} a_{\mathrm{FF}}^{C}\right) u_{i, \mathrm{P}},
$$

where $S_{\mathrm{P}}$ is added to the central coefficient, Eq. (29), and an extra LUDS source term $S_{u_{i}-\text { LUDS }}$ is added to the total source term of Eq. (30).

As a result of the application of the LUDS scheme to the convective terms of the stress equation, their $a_{\mathrm{F}}^{\tau}$ coefficients are also modified. They are equal to the LUDS convective coefficients for momentum, $a_{\mathrm{F}}^{C}$ given by Eq. (36a), multiplied by $\lambda / \rho$, and similarly for the $a_{\mathrm{FF}}^{C}$ coefficient, whose terms are dealt with as in the momentum equation.

\subsection{Formulation of the mass flow rates}

The mass flow rates $\left(\mathrm{F}_{\mathrm{f}}\right)$ appearing in the coefficients $a_{\mathrm{F}}^{C}$ and $a_{\mathrm{FF}}^{C}$ (Eq. (28) for UDS and Eq. (36a) and Eq. (36b) for LUDS) and in the mass conservation Eq. (16) are expressed in terms of the velocities at cell faces $\left(\tilde{u}_{i, \mathrm{f}}\right)$, which in turn need to be specified in terms of the cell centre values. The mass conservation equation is required to compute the pressure field, after obtaining the velocity field from the momentum balance, and this is traditionally accomplished via a staggered grid configuration. With this configuration the locations where the velocity and the pressure are computed are staggered by half a cell width, and this results in the appearance of nodal values while forcing mass conservation, thus ensuring a full coupling between those two quantities [4]. 
Since one of the main goals of the present work was the adoption of the non-staggered grid approach, a special velocity interpolation scheme, originally designed by Rhie and Chow [1], is required. In this work the formulation of Issa and Oliveira [15] for a time-marching algorithm is followed. Let us define for simplicity

$$
H\left(u_{i}\right) \equiv \sum_{\mathrm{F}} a_{\mathrm{F}} u_{i, \mathrm{~F}}
$$

and lets us single the pressure term out of the source term so that $S_{u_{i}}=-\sum_{l=1}^{3} B_{l i}^{\mathrm{P}}[\Delta p]_{l}^{\mathrm{P}}+S_{u_{i}}^{\prime}$. The momentum equations at nodes $\mathrm{P}$ and $\mathrm{F}$ can now be written as

$$
\begin{aligned}
& a_{P} u_{i, \mathrm{P}}=H\left(u_{i}\right)-\sum_{l=1}^{3} B_{l i}^{\mathrm{P}}[\Delta p]_{l}^{\mathrm{P}}+S_{u_{i}}^{\prime}+\left(\frac{\rho V}{\delta t}\right)_{\mathrm{P}} u_{i, \mathrm{P}}^{o}, \\
& a_{\mathrm{F}} u_{i, \mathrm{~F}}=H_{\mathrm{F}}\left(u_{i}\right)-\sum_{l=1}^{3} B_{l i}^{\mathrm{F}}[\Delta p]_{l}^{\mathrm{F}}+S_{u_{i} \mathrm{~F}}^{\prime}+\left(\frac{\rho V}{\delta t}\right)_{\mathrm{F}} u_{i, \mathrm{~F}}^{o},
\end{aligned}
$$

where the pressure differences are evaluated at cell center (i.e. $[\Delta p]_{l}^{\mathrm{P}}=p^{l^{+}}-p^{l^{-}}$, see Fig. 2).

According to Rhie and Chow's special interpolation method, the cell face velocity $\tilde{u}_{\mathrm{F}}$ is calculated by linear interpolation of the momentum equations, with exception of the pressure gradient which is evaluated as in the staggered approach. In [15] this idea is applied as

$$
\bar{a}_{P} \tilde{u}_{i, \mathrm{f}}=\overline{H\left(u_{i}\right)}+\overline{S_{u_{i}}^{\prime}}-B_{f i}^{\mathrm{f}}[\Delta p]_{f}^{\mathrm{f}}-\sum_{l \neq f}^{2} \overline{B_{l i}[\Delta p]_{l}}+\overline{\left(\frac{\rho V}{\delta t}\right)_{\mathrm{P}}} \tilde{u}_{i, \mathrm{f}}^{o},
$$

where the overbar denotes an arithmetic mean of quantities pertaining to $\mathrm{F}$ and $\mathrm{P}\left(\bar{u}_{\mathrm{f}}=0.5\left(u_{p}+\right.\right.$ $\left.u_{f}\right)$ ) cells. Notice that the pressure difference along direction $l=f$ is now evaluated at cell-face (i.e. $[\Delta p]_{f}^{\mathrm{f}}=p_{\mathrm{F}}-p_{\mathrm{P}}$ ), whereas the pressure at cell faces pertaining to $l \neq f$ pressure differences are calculated by linear interpolation of nodal values of pressure. In this way, the velocity at face $\mathrm{f}$ is now directly linked to pressures calculated at neighbour cell centres, as in the staggered arrangement.

By subtracting Eq. (42) from the averaged momentum equation resulting from the arithmetic average of all terms of Eqs. (40) and (41), the following final face-velocity equation is obtained:

$$
\tilde{u}_{i, \mathrm{f}}=\frac{\overline{a_{\mathrm{P}} u_{i, \mathrm{P}}}+\overline{B_{f i}^{\mathrm{P}}[\Delta p]_{f}^{\mathrm{P}}}-B_{f i}^{\mathrm{f}}[\Delta p]_{f}^{\mathrm{f}}+\overline{\left(\frac{\rho V}{\delta t}\right)_{\mathrm{P}}} \tilde{u}_{i, \mathrm{f}}^{o}-\overline{\left(\frac{\rho V}{\delta t} u_{i}^{o}\right)_{\mathrm{P}}}}{\overline{a_{\mathrm{P}}}},
$$

which avoids the need to compute and store the $H$-operator. This expression is replaced in Eq. (16) to get the convective fluxes without the necessity to store each component of the face velocities at the present and previous time level, thus greatly reducing the storage requirements.

\subsection{Formulation of the cell-face stresses}

In the momentum equation, it is necessary to compute the stresses at cell faces from stress values at cell centres and there is a stress-velocity coupling problem, akin to the pressure-velocity coupling, that needs to be properly solved. If a linear interpolation of cell centered values of 


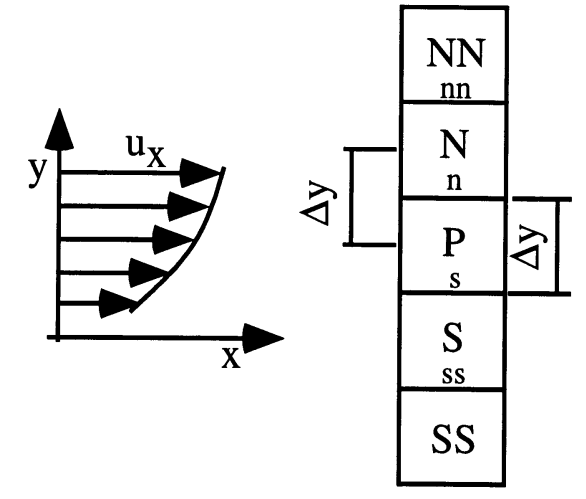

Fig. 4. An uniform grid representing cell $\mathrm{P}$ and its neighbours in the north-south vicinity.

stress is used to compute face values, a possible lack of connectivity between the stress and velocity fields may result, even with Newtonian fluids, as shown below in an example taken from a simplified $x$-momentum equation.

- Example

Consider the equally-spaced grid of Fig. 4 and the discretised form of the term $\partial \tau_{x y} / \partial y$ in Cartesian coordinates, which is

$$
\left(\frac{\partial \tau_{x y}}{\partial y}\right)_{\mathrm{P}}=\frac{\tau_{x y, \mathrm{n}}-\tau_{x y, \mathrm{~s}}}{\Delta y}=\frac{1}{\Delta y}\left[\frac{\tau_{x y, \mathrm{~N}}+\tau_{x y, \mathrm{P}}}{2}-\frac{\tau_{x y, \mathrm{P}}+\tau_{x y, \mathrm{~S}}}{2}\right]=\frac{1}{2 \Delta y}\left[\tau_{x y, \mathrm{~N}}-\tau_{x y, \mathrm{~S}}\right] .
$$

Assuming for simplicity a fully developed flow and only the $u_{x}$ contribution to the Newtonian shear stress $\left(\tau_{x y}=\eta \partial u_{x} / \partial y\right.$, ) the stresses in Eq. (44) can be expressed in terms of nodal values of the velocity component $u_{x}$. Considering that $\tau_{x y, N}$ depends on $u_{x, n n}$ and $u_{x, n}$, a little algebra leads to

$$
\left(\frac{\partial \tau_{x y}}{\partial y}\right)_{\mathrm{P}}=\frac{\eta}{4 \Delta y^{2}}\left[u_{x, \mathrm{NN}}-2 u_{x, \mathrm{P}}+u_{x, \mathrm{SS}}\right]
$$

Instead of the above, in the Newtonian Navier-Stokes equation the corresponding term is usually written as $\eta \partial^{2} u_{x} / \partial y^{2}$, and standard central differencing gives

$$
\begin{aligned}
& \left(\frac{\partial \tau_{x y}}{\partial y}\right)_{\mathrm{P}}=\eta \frac{\partial^{2} u_{x}}{\partial y^{2}}=\frac{\eta}{\Delta y}\left[\left(\frac{\partial u_{x}}{\partial y}\right)_{\mathrm{n}}-\left(\frac{\partial u_{x}}{\partial y}\right)_{\mathrm{s}}\right]=\frac{\eta}{\Delta y}\left[\frac{u_{\mathrm{x}, \mathrm{N}}-u_{\mathrm{x}, \mathrm{P}}}{\Delta y}-\frac{u_{\mathrm{x}, \mathrm{P}}-u_{\mathrm{x}, \mathrm{S}}}{\Delta y}\right]= \\
& \frac{\eta}{\Delta y^{2}}\left[u_{\mathrm{x}, \mathrm{N}}-2 u_{\mathrm{x}, \mathrm{P}}+u_{\mathrm{x}, \mathrm{S}}\right],
\end{aligned}
$$

a well-known result in CFD.

In conclusion, whereas in the Newtonian CFD approach there is a strong coupling between the stress at node $\mathrm{P}$ and the velocities in the near-neighbour nodes (Eq. (46)), that is not the case in the generalised treatment of the stress tensor (Eq. (45)), where a physically unrealistic checkerboard velocity field pattern can result in the correct stress field, if a linear interpolation scheme is used for calculating stresses at cell faces. 
To remedy this anomaly, stress values at a cell face, like in Eq. (44) above, must not be obtained by linear interpolation of stress values at neighbouring cells but ought to be based on the novel interpolation method developed in this work and now explained. The discretised stress Eq. (32) is re-written as

$$
a_{\mathrm{P}}^{\tau} \tau_{i j, \mathrm{P}}=H\left(\tau_{i j}\right)+\sum_{l}^{3}\left(b_{l i}\left[\Delta u_{j}\right]_{l}^{\mathrm{P}}+b_{l j}\left[\Delta u_{i}\right]_{l}^{\mathrm{P}}\right)+S_{\tau_{i j}}^{\prime},
$$

where the $H$-operator is defined as before, in Eq. (39) and terms proportional to velocity differences were made to appear explicitly with help of the definition

$$
b_{l i}=\eta B_{l i}+\lambda \sum_{k}^{3} B_{l k} \tau_{i k} \text {. }
$$

When a stress component $\tau_{i j}$ is required at a cell face $\mathrm{f}$, in the momentum equation, it is obtained by arithmetic averaging the stress equations written for cell P (Eq. (47)) and for its neighbour $\mathrm{F}$ across face $\mathrm{f}$, with the exception that velocity differences straddling the face are to be evaluated directly. Thus, the spirit of Rhie and Chow [1] interpolation for the face velocity is followed, guarantying a good connection between a face stress and the velocity values at either side of the face. This procedure is equivalent to defining the cell-face stress as (compare with Eq. $(42))$ :

$$
\overline{a_{\mathrm{P}}^{\tau}} \tilde{\tau}_{i j, f}=\overline{H\left(\tau_{i j}\right)}+\overline{b_{f j}}\left[\Delta u_{i}\right]_{f}^{\mathrm{f}}+\overline{b_{f i}}\left[\Delta u_{j}\right]_{f}^{\mathrm{f}}+\left(\overline{\sum_{l \neq f}^{2} b_{l j}\left[\Delta u_{i}\right]_{l}^{\mathrm{P}}+\sum_{l \neq f}^{2} b_{l i}\left[\Delta u_{j}\right]_{l}^{\mathrm{P}}}\right)+\overline{S_{\tau_{i j}}^{\prime}}
$$

where it is noted that the terms proportional to velocity differences not aligned with the face direction, i.e. $\left[\Delta u_{i}\right]_{l \neq f}^{\mathrm{P}}$, are evaluated by arithmetic averaging, like the remaining source term $S_{\tau_{i j}}^{\prime}$ and the $H$-term. Instead of using Eq. (49) to evaluate a stress at a cell face, it is computationally more efficient to follow an incremental approach, in which the cell-face stress of Eq. (49) is subtracted from an average cell centered stress obtained from Eq. (47) applied to both nodes P and $\mathrm{F}$, as previously carried out for velocity, to yield

$$
\tilde{\tau}_{i j, \mathrm{f}}=\frac{\overline{a_{\mathrm{P}}^{\tau} \tau_{i j}}+\bar{b}_{f j}\left[\Delta u_{i}\right]_{f}^{\mathrm{f}}+\bar{b}_{f i}\left[\Delta u_{j}\right]_{f}^{\mathrm{f}}-\left(\overline{b_{f j}\left[\Delta u_{i}\right]_{f}+b_{f i}\left[\Delta u_{j}\right]_{f}}\right)}{\overline{a_{P}^{\tau}}} .
$$

It is clear from this equation that the stress at face $\mathrm{f}\left(\tilde{\tau}_{i j \mathrm{f}, \mathrm{f}}\right)$ is now directly coupled to the nearby velocities through the term in $\left[\Delta u_{i}\right]_{f}^{\mathrm{f}} \equiv u_{i, \mathrm{~F}}-u_{i, \mathrm{P}}$; in fact Eq. (50) applied to the simplified situation of the previous example leads exactly to the 'good' formulation Eq. (46) and not to the un-coupled formulation (Eq. (45)), demonstrating that the decoupling stress-velocity question is well addressed. In [5], it is shown that the approximation implied by Eq. (50) is equivalent to a term in the fourth derivative of the stress component and thus, this smoothing term does not contribute to artificial diffusion.

\subsection{The solution algorithm}

As in any velocity correction procedure [16], pressure is calculated indirectly from the restraint imposed by continuity, since the momentum equation, which explicitly contains a pressure gradient term, is used to compute the velocity vector. Improvements to the SIMPLE procedure 
of Patankar and Spalding have since been developed and the SIMPLEC algorithm of Van Doormal and Raithby [17] has been here adopted and extended to cope with the stress equation. This algorithm was originally developed for iterative marching, however, the time-marching version explained in [15] is used instead. Time marching allows for the solution of transient flows, with the added advantage that it can be used in steady flows as an alternative to implement under-relaxation. For this reason, the equations have been written, from the outset, with the time-dependent terms included (see Eqs. (9) and (10)).

The presence of the constitutive equation produces little changes on the original SIMPLEC method, which is mainly concerned with the calculation of pressure from the continuity equation. Two new steps are introduced in the initial part of the algorithm to account for the stress equation, as follows:

- The stress field results from solution of the six implicit constitutive equations, which is carried out prior to the first time the three momentum equations are handled;

- Subsequently, the momentum equations are solved implicitly for each velocity component, with stresses from the step above being included into the source term. The important point here is to base the divergence term (Eq. (24)) on the specially developed interpolation method defined by Eq. (50).

An overview of the solution algorithm is now given. For each time advancement, $\delta t$, three steps are required. In the first step, the six stress components are obtained sequentially from the implicit constitutive equation (from Eq. (32), with the $\tau_{i j, \mathrm{P}}^{0}$ term included in the source):

$$
a_{\mathrm{P}}^{\tau} \tau_{i j, \mathrm{P}}^{*}-\sum_{\mathrm{F}} a_{\mathrm{F}}^{\tau} \tau_{i j, \mathrm{~F}}^{*}=S_{\tau_{i j}}
$$

where the coefficients and source term are based on the previous time-level velocity and stress, and $\tau_{i j}^{*}$ denotes the new time-level of $\tau_{i j}$. Eq. (51) represents a set of linear equations to be solved for $\tau_{i j}^{*}$.

In the second step, the momentum equations (27) are solved implicitly for each velocity component:

$$
\left(\sum_{\mathrm{F}} a_{\mathrm{F}}+S_{\mathrm{P}}+\frac{\rho V_{\mathrm{P}}}{\delta t}\right) u_{i, \mathrm{P}}^{*}-\sum_{\mathrm{F}} a_{\mathrm{F}} u_{i, \mathrm{~F}}^{*}=\sum_{l}^{3} B_{l i}\left[\Delta p^{o}\right]_{l}^{\mathrm{P}}+S_{u_{i}}^{\prime}
$$

where the pressure gradient term is based on previous time-level pressure values and has been singled out of the remaining source term for later convenience. The stress-related source term (Eq. (24)) is based on newly obtained cell-face stress $\tilde{\tau}_{i j}^{* f}$, calculated from Eq. (50), which requires the central coefficient of the stress equation $\left(a_{\mathrm{P}}^{\tau}\right)$. This is why the stress equation should be solved before the momentum equation.

Starred velocity components $\left(u_{i}^{*}\right)$ do not generally satisfy the continuity equation. The third step of the algorithm involves a correction to $u_{i}^{*}$, so that an updated velocity field $u_{i}^{* *}$ will satisfy both the continuity equation and the following split form of the momentum equation:

$$
\left(\sum_{\mathrm{F}} a_{\mathrm{F}}+S_{\mathrm{P}}\right) u_{i, \mathrm{P}}^{*}+\frac{\rho V_{P}}{\delta t} u_{i, \mathrm{P}}^{* *}=\sum_{\mathrm{F}} a_{\mathrm{F}} u_{i, \mathrm{~F}}^{*}-\sum_{l} B_{l i}\left[\Delta p^{*}\right]_{l}^{\mathrm{P}}+S_{u_{i}}^{\prime}
$$

Note here that only the time-dependent term is updated to the new time level $u_{i}^{* *}$, a feature of the SIMPLEC algorithm. Subtraction of this equation from Eq. (52) and forcing the $u_{i}^{* *}$ field 
to satisfy continuity $\left(\sum_{\mathrm{f}} F_{\mathrm{f}}^{* *}=0\right.$, see Eqs. (16) and (17)) leads to the pressure and velocity correction equations $\left(p^{\prime} \equiv p^{*}-p^{0}\right)$ :

$$
\begin{aligned}
& a_{\mathrm{P}}^{p} p_{\mathrm{P}}^{\prime}=\sum_{\mathrm{F}} a_{\mathrm{F}}^{p} p_{\mathrm{F}}^{\prime}-\sum_{\mathrm{f}} F_{\mathrm{f}}^{*} \quad\left(a_{\mathrm{P}}^{p}=\sum_{\mathrm{F}} a_{\mathrm{F}}^{\mathrm{p}} ; \quad a_{\mathrm{F}}^{p}=\frac{\rho B_{\mathrm{f}}^{2}}{(\overline{\rho V / \delta t})_{\mathrm{f}}}\right), \\
& \frac{\rho V_{\mathrm{P}}}{\delta t}\left(u_{i}^{* *}-u_{i}^{*}\right)_{\mathrm{P}}=-\sum_{l} B_{l i}^{\mathrm{P}}\left[\Delta p^{\prime}\right]_{l}^{\mathrm{P}},
\end{aligned}
$$

which completes the algorithm.

The various sets of algebraic equations are solved with either a symmetric or a bi-conjugate gradient method for the pressure and the remaining variables, respectively [18]. In both cases, the matrices are pre-conditioned by an incomplete LU decomposition.

\subsection{Boundary conditions}

Boundary conditions need to be set for the velocity components at inlets, outlets, symmetry planes and walls. The stress equations are hyperbolic thus, a boundary condition is only required at the inlet. For incompressible fluids, the absolute value of pressure is irrelevant and only its variation matters. It is the usual practice to set the pressure to zero at a particular cell in the calculation domain, such as at the inlet, and to set initially the pressure field to zero. The pressure field will then be corrected in each iteration to force local mass conservation, according to the algorithm just described.

- Inlet: At the inlet boundary face, velocity and stress components are given according to some pre-specified profile (from theory or from measured data). In the present problems, the streamwise velocity component was set equal to a uniform constant value and all other quantities were set to zero.

- Outlet: The outlet is located far from the region of interest, where the flow is locally parabolic. Thus, zero streamwise gradients are assumed and numerically, this is accomplished by setting values of the velocity and stress at the outlet cell faces equal to those at the corresponding upstream cell centres. The same applies to pressure gradients, which is equivalent to performing a linear extrapolation of pressure from the two internal cells to the outlet boundary face. It is also necessary to adjust the velocities at these boundary faces so that overall mass conservation is satisfied.

- Symmetry planes: Across a symmetry plane, the convective and diffusive fluxes must vanish. These two conditions were applied to all variables using reflection rules in fictitious symmetric cells (Fig. 5) and result in the following set of boundary conditions [19]). The velocity component $u_{i}$ at the boundary $\mathrm{f}$ is calculated by linear interpolation from their counterparts at $\mathrm{P}$ and $\mathrm{P}^{\prime}$, the cells on both sides of the symmetry plane (Fig. 5), giving

$$
u_{i, \mathrm{f}}=u_{i, \mathrm{P}}-u_{n, \mathrm{P}} n_{i} \quad \text { with } \quad u_{n, \mathrm{P}}=\sum_{j} u_{j, \mathrm{P}} n_{j},
$$

where $u_{n, \mathrm{P}}$ is the component of the velocity vector normal to the symmetry plane and $n_{i}$ is the $i$-component of the unit vector normal to the symmetry plane.

For any scalar quantity, such as pressure, the reflexion rule at symmetry planes leads to 


$$
p_{\mathrm{f}}=p_{\mathrm{P}}
$$

and for the stress field $\left(\tau_{i j}\right)$ or the corresponding traction vector $\left(T_{i}=\sum_{j} \tau_{i j} n_{j}\right)$ gives

$$
T_{i, \mathrm{f}}=T_{i, \mathrm{P}} \Leftrightarrow \sum_{k} \tau_{i k, \mathrm{f}} n_{k}=\left(\sum_{k} \sum_{j} \tau_{j k, \mathrm{P}} n_{j} n_{k}\right) n_{i} \equiv T_{n, \mathrm{P}} n_{i}
$$

which is an implicit set of equations on the unknown stress components at the boundary face $\left(\tau_{i k, \mathrm{f}}\right)$. However, not each individual component of the stress tensor is required at $\mathrm{f}$ as deduced from inspection of the momentum equations. Indeed, it is seen from Eq. (24) that the contribution from face $\mathrm{f}$ to the total stress source in cell $\mathrm{P}$ is just

$$
\left(S_{u_{i}-\text { stress }}\right)_{\mathrm{f}}=\sum_{j} B_{f j}^{\mathrm{f}} \tau_{i j, \mathrm{f}}=B_{\mathrm{f}} \sum_{j} \tau_{i j, \mathrm{f}} n_{j},
$$

where the unit normal vector is computed as $n_{j}=B_{f j} / B_{\mathrm{f}}$. Thus, the boundary condition (57) at the symmetry plane face $\mathrm{f}$ is implemented as

$$
\left(S_{u_{i}-\text { stress }}\right)_{\mathrm{f}}=T_{n, \mathrm{P}} B_{\mathrm{f}} n_{i} \text {. }
$$

- Wall: Boundary conditions for the velocity field are easy to impose. For a wall moving at velocity $u_{\mathrm{w}}$, the usual no slip condition for the components component $u_{i}$, of the velocity vector is simply

$$
u_{i, \mathrm{f}}=u_{i, w} .
$$

As far as the pressure is concerned, its value at the wall is linearly extrapolated from the two nearest neighbour cells, the method used in standard CFD [5].

Due to the hyperbolic nature of the constitutive equations, there is no need to explicitly impose boundary conditions for the stresses and thus the constitutive equations must in principle be solved as part of the problem to obtain stress values at the walls. However, to reduce computational time approximate wall boundary conditions were imposed. As a first attempt, the stresses were linearly extrapolated to the wall face, a procedure inspired by the method used for pressure. This proved to be highly unstable, a possible consequence of the steep local gradients in the stress boundary-layer which develops along the wall, and consequently

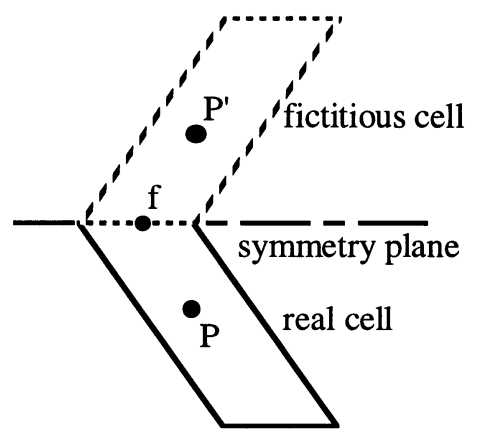

Fig. 5. The fictitious cell adjacent to a symmetry plane. 
wall boundary conditions for stress were obtained directly from the constitutive equations. For a wall situated at a general cell face f, the constitutive Eq. (10) is reduced to

$$
J_{\mathrm{f}} \tau_{i j, \mathrm{f}}=\eta_{\mathrm{f}}\left[\beta_{f j} \frac{\partial u_{i}}{\partial \xi_{f}}+\beta_{f i} \frac{\partial u_{j}}{\partial \xi_{f}}\right]+\lambda_{\mathrm{f}}\left[\left(\beta_{f k} \tau_{k j}\right) \frac{\partial u_{i}}{\partial \xi_{f}}+\left(\beta_{f k} \tau_{k i}\right) \frac{\partial u_{j}}{\partial \xi_{f}}\right]-\frac{2}{3} \eta_{\mathrm{f}} \beta_{f k} \frac{\partial u_{k}}{\partial_{f}} \delta_{i j},
$$

after setting the convective terms to zero and realizing that velocity differences in the wall face must vanish (i.e. $\left.\left(\partial u_{j} / \partial \xi_{l}\right)_{l \neq f}=0\right)$. Further simplification of Eq. (61) leads to analytical expressions for the stress components at the wall, however, this causes localised oscillations in the numerical approximation. After numerical experiments, it has been decided to solve the implicit Eq. (61) for the wall face stresses $\tau_{i j, \mathrm{f}}$ by incorporating it into the overall iterative procedure. Therefore, the stresses appearing on the right-hand-side of Eq. (61) lag by a time level and, as convergence is attained, they must tend to the stress value on the left-hand-side. This approach proved to be much more accurate than the approach using analytical expressions for the stress values at the wall.

\section{Numerical results and discussion}

The algorithm was applied to the solution of two different flow problems of upper convected Maxwell fluids. First, the entry flow considered by Eggleton et al. [2] was investigated in detail with both orthogonal and non-orthogonal grids. Later, the flow past a circular cylinder of Huang and Feng [20] was selected for assessing the performance of the algorithm in a more complex, non-orthogonal geometry.

\subsection{Entry flow: flow conditions and convergence issues}

Although [2] presents the equations for an Oldroyd-B model, their calculations of the entry flow problem were carried out for a fluid without a Newtonian solvent contribution, i.e. their constitutive model only had a polymer contribution from an elastic dumbbell model which is equivalent to the UCM equation. The imposed uniform inlet velocity $\left(U_{\text {in }}\right)$ and the half-channel height $(H)$, together with the fluid characteristics, defined the following base Deborah (De) and Reynolds (Re) numbers:

$$
\mathrm{De}=\frac{\lambda U_{\text {in }}}{H}=0.1 \quad \text { and } \quad \mathrm{Re}=\frac{\rho U_{\text {in }} H}{\eta}=20 .
$$

Whenever required, variation of the Deborah and Reynolds numbers was carried out with changes in the fluid time constant $(\lambda)$ and the viscosity $(\eta)$, respectively.

The two dimensional geometry is represented in Fig. 6, where the domain size, the nomenclature used for the mesh size and the coordinate system are defined. For purposes of mesh generation the geometry was divided into two blocks, with block 1 in the wall-free region having $\mathrm{NX}_{1}$ by $\mathrm{NY}$ cells, patched to block 2 in the wall region with $\mathrm{NX}_{2}$ by $\mathrm{NY}$ cells.

In order to assess the grid-size effect, various orthogonal grids were defined, however results pertaining to only three of them (meshes 3,5 and 7) are presented here in order to avoid cluttering of the figures. The characteristics of all grids are briefly presented in Table 1, where 


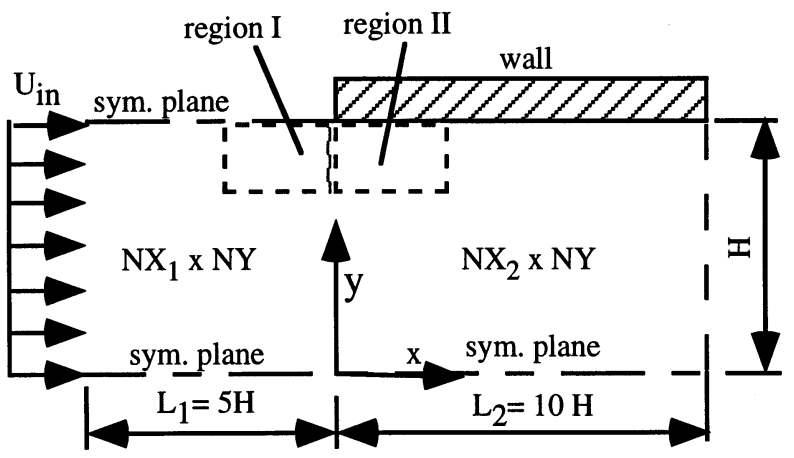

Fig. 6. Schematic representation of the slip-stick geometry.

$f_{x}$ end $f_{y}$ are geometrical factors used to concentrate nodes close to the wall leading edge. Special care was taken to ensure that the mesh size varied smoothly over the flow domain.

The performance of the algorithm with a non-orthogonal grid, shown in Fig. 7, was also assessed with this entry flow. This particular flow is very suitable for this numerical experiment as it allows the use of various types of grid. The non-orthogonal mesh was based on the same number of blocks and cells as the orthogonal mesh 7, and was obtained by skewing the cells by $30^{\circ}$ relative to the $y$-direction. Thus, although Fig. 7 is a plot of the non-orthogonal grid in the vicinity of the wall discontinuity, it also provides a good idea of the finest orthogonal grid, mesh 7. As expected, the results obtained with the non-orthogonal mesh were identical to those calculated with the orthogonal mesh and a comparison is presented in Section 4.4.

Fig. 8(a-d) show local details, around the geometric singularity, of longitudinal profiles of the stress along a line close to the symmetry plane and wall $(y / H=0.985)$, normalised according to

$$
T_{i j}=\frac{\tau_{i j}}{\frac{\eta U_{\mathrm{in}}}{H}} .
$$

Table 1

Summary of the main characteristics of the grids

\begin{tabular}{|c|c|c|c|c|c|c|c|c|}
\hline \multirow[t]{2}{*}{ Grid } & \multicolumn{4}{|l|}{ Block 1} & \multicolumn{4}{|l|}{ Block 2} \\
\hline & $\mathrm{NX}_{1} \times \mathrm{NY}$ & $f_{x}$ & $f_{y}$ & $\delta_{x, \min }^{*} / \delta_{y, \min }^{*}$ & $\mathrm{NX}_{2} \times \mathrm{NY}$ & $f_{x}$ & $f_{y}$ & $\delta_{x, \min }^{*} / \delta_{y, \min }^{*}$ \\
\hline Mesh 1 & $20 \times 20$ & 0.9131 & 0.9500 & $0.1 / 0.03$ & $40 \times 20$ & 1.0422 & 0.9500 & $0.1 / 0.03$ \\
\hline Mesh 2 & $20 \times 20$ & 0.8712 & 0.9500 & $0.05 / 0.03$ & $40 \times 20$ & 1.0701 & 0.9500 & $0.05 / 0.03$ \\
\hline Mesh 3 & $30 \times 20$ & 0.9085 & 0.9500 & $0.03 / 0.03$ & $60 \times 20$ & 1.0486 & 0.9500 & $0.03 / 0.03$ \\
\hline Mesh 4 & $30 \times 40$ & 0.9085 & 0.9595 & $0.03 / 0.01$ & $60 \times 40$ & 1.0486 & 0.9595 & $0.03 / 0.001$ \\
\hline Mesh 5 & $50 \times 40$ & 0.9293 & 0.9595 & $0.01 / 0.01$ & $80 \times 40$ & 1.0505 & 0.9595 & $0.01 / 0.01$ \\
\hline Mesh 6 & $50 \times 60$ & 0.9293 & 0.9657 & $0.01 / 0.005$ & $80 \times 60$ & 1.0505 & 0.9657 & $0.01 / 0.005$ \\
\hline Mesh 7 & $70 \times 60$ & 0.9428 & 0.9657 & $0.005 / 0.005$ & $100 \times 60$ & 1.0465 & 0.9657 & $0.005 / 0.005$ \\
\hline
\end{tabular}

$\delta_{x}^{*}, \delta_{y}^{*}$ are the cell dimensions normalised by the channel half-height. 


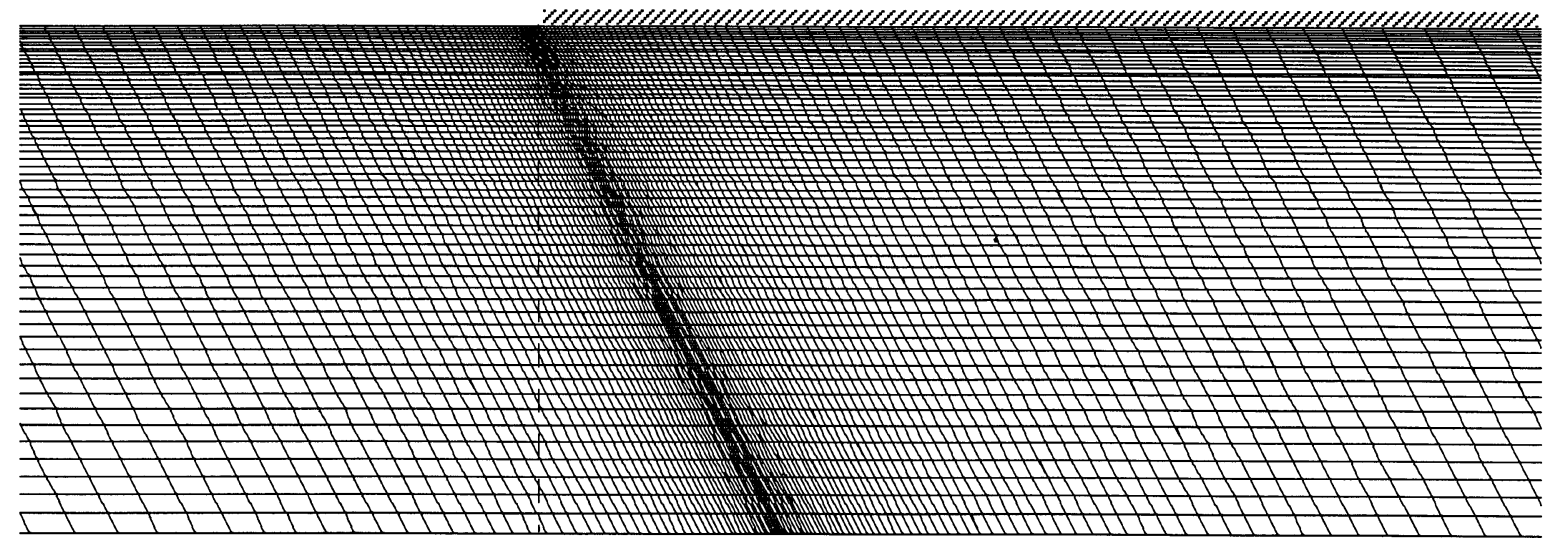

Fig. 7. The non-orthogonal grid based on the orthogonal mesh 7 for the slip-stick geometry. The skewness of the cells is $30^{\circ}$ relative to the $y$-direction (zoom in the region $x / H=-1-+2$ ).

The main feature of the figures is the large peak in all components of the stress as the flow approaches the wall. This feature is also found in the reverse problem, the usual stick-slip flow, for which many authors give profiles similar to those of Fig. 8 but often with incipient or strong oscillations in the stress values near the singularity [21]. In terms of grid refinement, Fig. 8 demonstrates that predictions based on mesh 3 are not yet grid independent, whereas the differences between the results computed with meshes 5 and 7 are only on the magnitude of the maximum values of the stress near the geometrical discontinuity. Far from the location of the slip-stick interface, there are no major differences between the various predictions and the flow development is independent of the mesh refinement, except for the three coarser grids of Table 1.

The current calculations were carried out with a grid much finer than those of [2] (their finest grid has a minimum spacing of $0.02 \mathrm{H}$ compared with $0.005 \mathrm{H}$ here). A comparison with their results close to the slip-stick boundary is difficult because of the large oscillations in their predictions. However, downstream of the discontinuity, at $x / H=0.6$, our calculations compare well with Eggleton et al., as shown in the lateral profiles of the stress components of Fig. 9. Although those profiles are located some distance downstream from the wall leading edge, it can still be seen that the predictions with mesh 3 are slightly different from those with meshes 5 and 7, indicating that mesh 3 is inadequate to yield mesh independent results.

Still further downstream from the wall leading edge, the flow tends to a fully developed situation and the predictions of $u / U_{\mathrm{in}}, T_{x x}$ and $T_{x y}$ at $x / H=10$ are indistinguishable from the theoretical profiles, thus giving some support to the correct implementation of the viscoelastic model in the finite-volume code. We may thus conclude that mesh 7 essentially yields mesh-independent results, but for the singular point, and that the method is validated against the independent results of [2].

With increased Deborah numbers, many more time steps (or iterations) are required to achieve a steady state solution, reflecting the so-called high Weissenberg number problem [22]. Thus, fine mesh results at high Deborah numbers require smaller time-steps and the first order accurate UDS scheme tends to be more effective in procuring iterative convergence. For finer 
$\mathrm{T}_{\mathrm{xx}}$

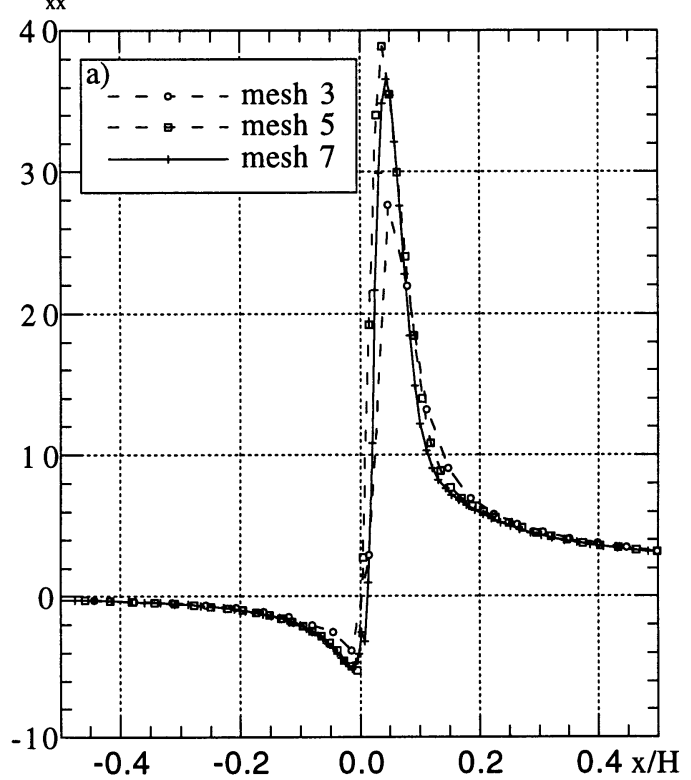

$\mathrm{T}_{\mathrm{xy}}$

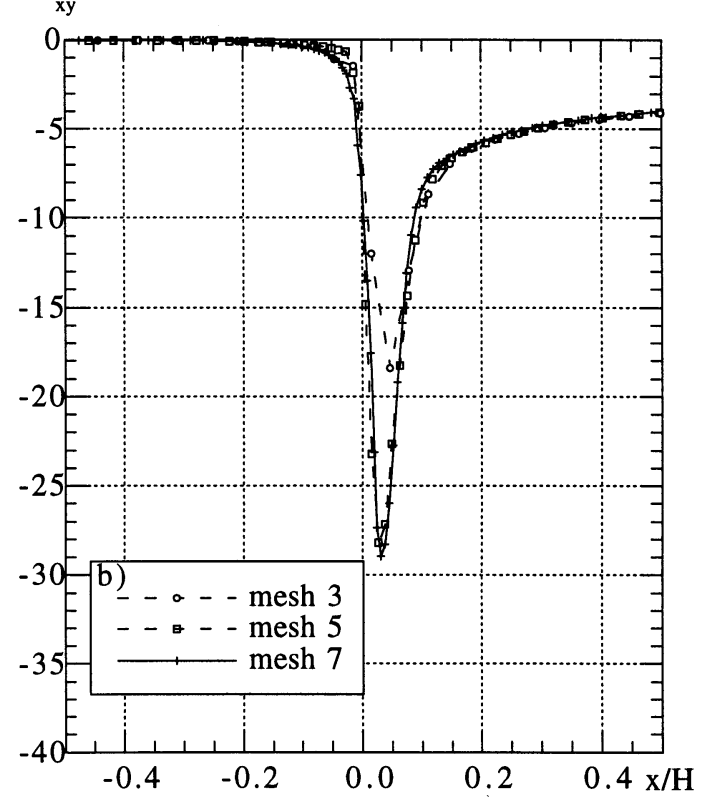

$\mathrm{T}_{\mathrm{yy}}$
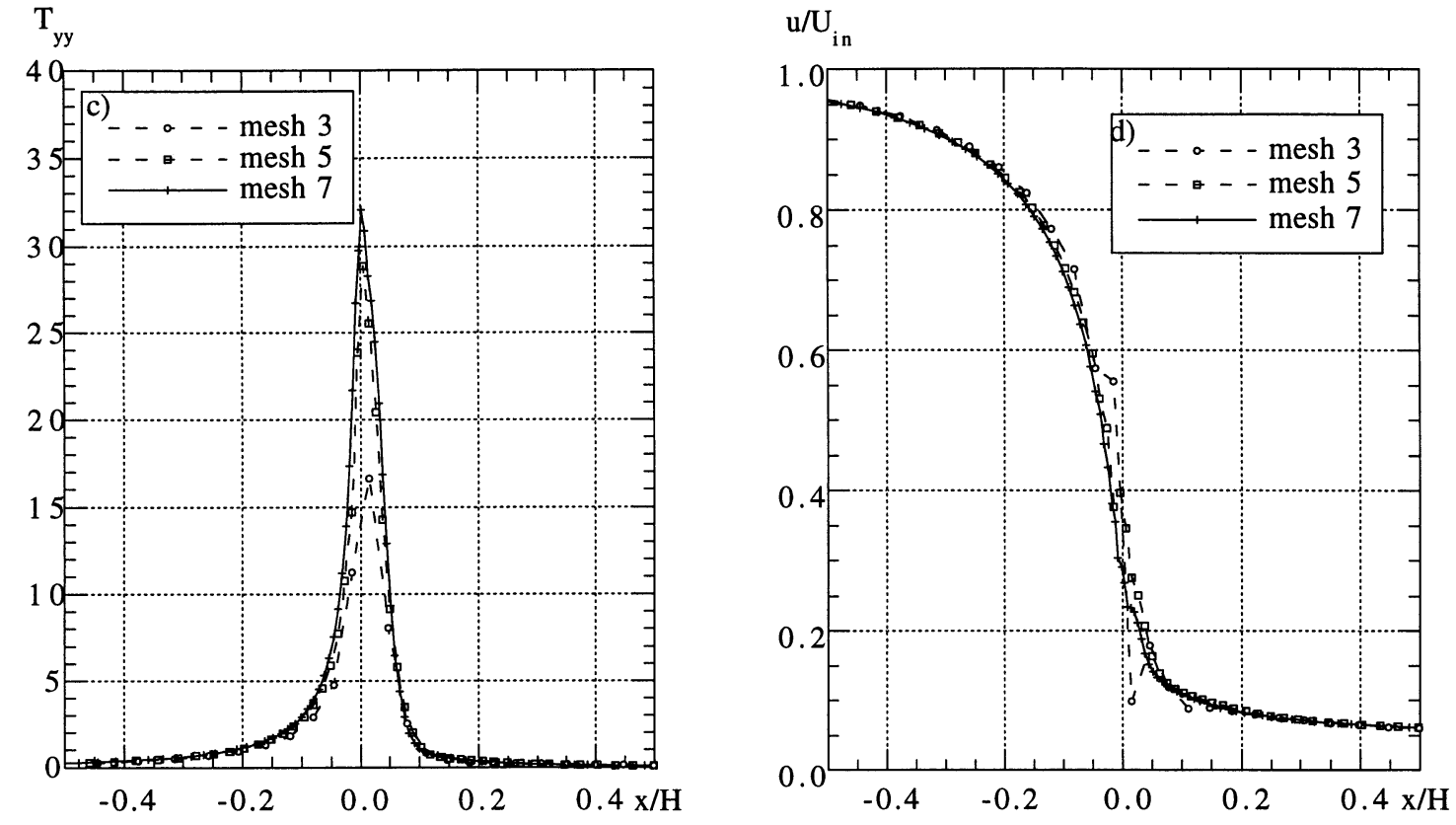

Fig. 8. Effect of grid refinement on longitudinal profiles near the wall at $y / H=0.985$ ( $\mathrm{De}=0.1, \operatorname{Re}=20, \mathrm{LUDS})$. (a) axial normal stress $\left(T_{x x}\right)$; (b) shear stress $\left(T_{x y}\right)$; (c) transversal normal stress $\left(T_{y y}\right)$; (d) axial velocity $\left(u / U_{\text {in }}\right)$. 

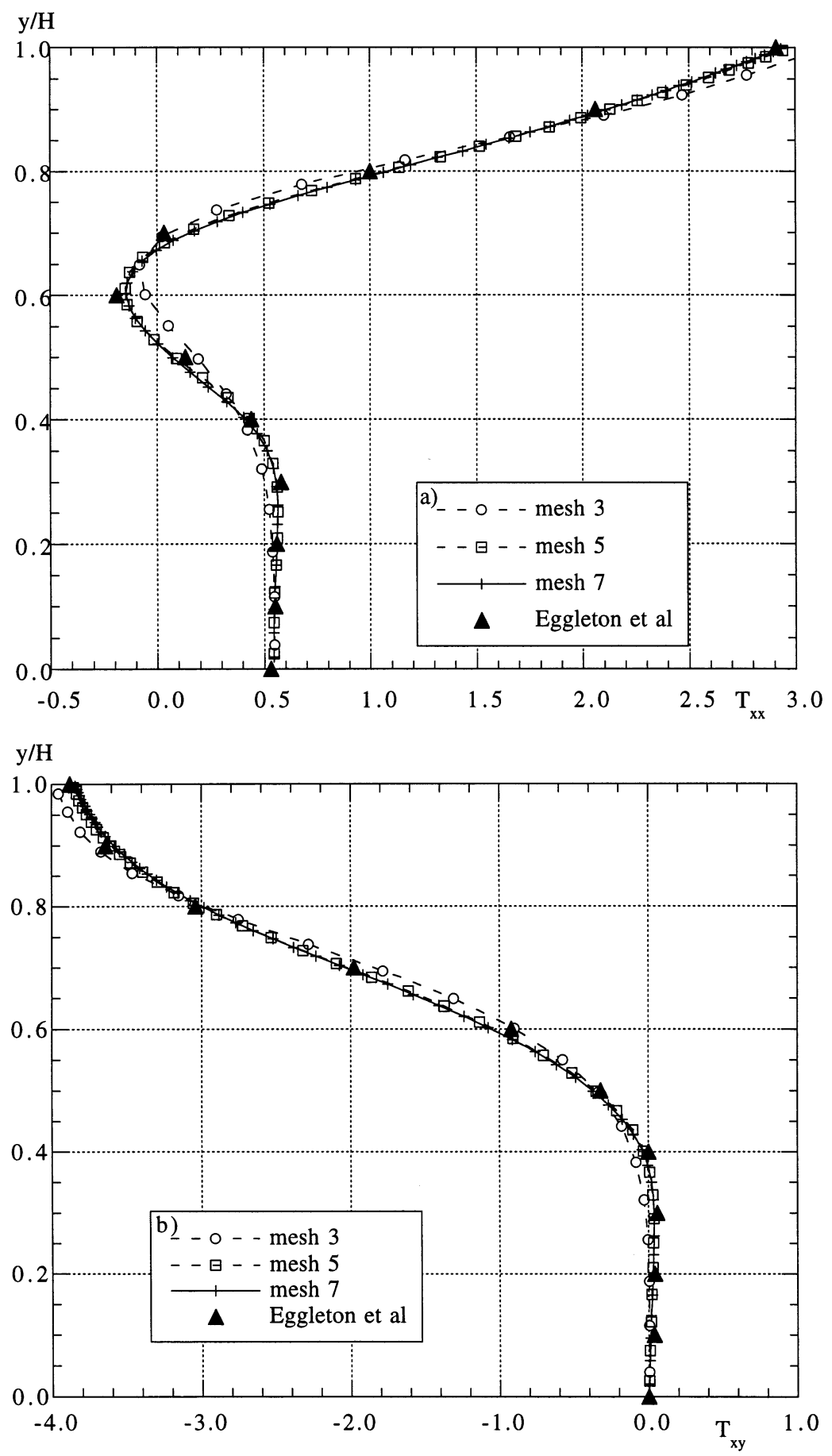

Fig. 9. Comparison between the current predictions and those of Eggleton et al. at $x / H=0.6(\mathrm{De}=0.1, \mathrm{Re}=20$, LUDS). (a) lateral profile of the longitudinal normal stress; (b) lateral profile of the shear stress. 


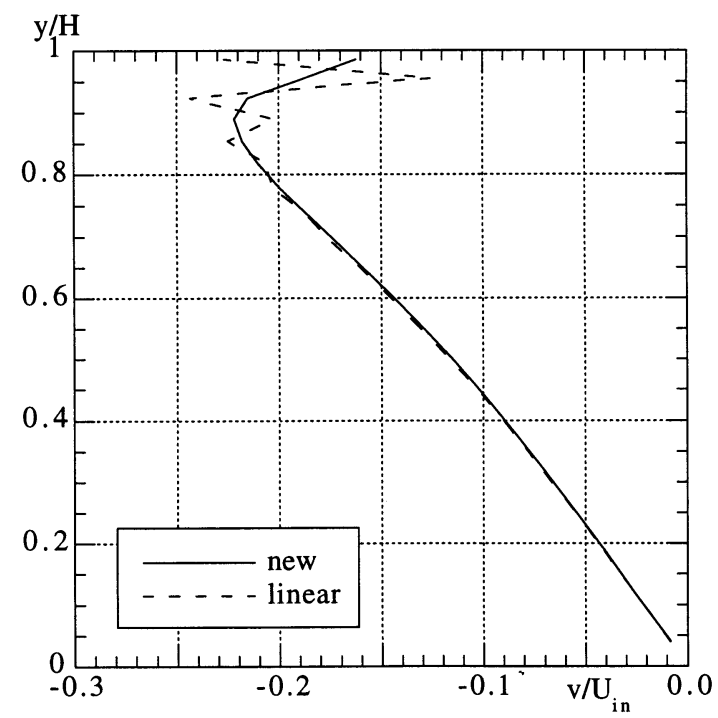

Fig. 10. Comparison of the lateral velocity components near the slip-stick junction $(x / H=0.015$, mesh 3$)$ based on the linear and the new interpolation schemes for the stresses.

grids, iterative convergence was also found to be more difficult: for mesh 7 , solutions could be obtained for Deborah numbers from 0.1 to 1.0 at a Reynolds number of 20, whereas for the coarser mesh 3, Deborah numbers in excess of 1.0 could be reached for the same Reynolds number, and Deborah numbers higher than 0.5 for a Reynolds number of 100 . These limiting values of the Deborah number are of the same order of magnitude as those found in the literature for the same flow geometry [2].

Attention is now turned to some numerical aspects of the proposed algorithm, namely to the question of velocity-stress decoupling. The novel method designed to obtain the cell-face stresses in the collocated mesh arrangement (Section 3.5) proved to be advantageous in more than one way. It coupled efficiently the velocity, pressure and stress fields, eliminating the oscillations found when the linear interpolation was used instead, as can be seen in the transverse profile of the lateral velocity component of Fig. 10. For the longitudinal velocity and stress tensor components, similar effects to those seen in Fig. 10 were observed. In addition, the use of this special interpolation scheme also speeded up iterative convergence in comparison to the linear scheme, with the decay of the residuals of the various equations being roughly four times faster, as shown in Fig. 11. Here, the residuals of the various algebraic equations are defined as the norm of the sum of all terms in the equations, when all the terms are on the same side of the equal sign. These residuals are suitably non-dimensionalized and should essentially tend to zero as time-marching proceeds. The criteria to stop time-marching, and assume converged fields, was for the largest residual to fall below $10^{-4}$, as seen in Fig. 11. 


\subsection{Entry flow: the Deborah number effect}

The effect of increasing the Deborah number upon the hydrodynamic characteristics of the flow was investigated in a comparison between the results of Newtonian $(\mathrm{De}=0)$ and viscoelastic flow cases, for Deborah numbers ranging from 0.1 to 1.0, computed in the finest orthogonal grid. The comparison will be carried out with streamline plots, as well as profiles and contours of the various stress and velocity components, with emphasis on the wall leading edge region.

The streamline plots of Fig. 12 show that for low Deborah numbers $(\mathrm{De}=0.1)$ the mean flow pattern is barely affected relative to the Newtonian flow case, however, differences are strongly enhanced for higher viscoelasticity, as in the Deborah number case of 1.0 (see streamlines in Fig. 12(b)). Two differences are worth mentioning in this respect: viscoelasticity causes a strong flow deceleration near the wall, in a layer extending to approximately $30 \% \mathrm{H}$, and by continuity the flow velocity increases in the central part of the channel; further downstream of the slip-stick interface the viscoelastic fluid accelerates in the wall region, a flow feature absent in the Newtonian fluid flow case. There is a third effect which can be just perceived in the comparison of Fig. 12(a) and in other profiles not shown here: for $\mathrm{De}=0.1$, the flow starts to deviate from the wall leading edge later than for the Newtonian fluid. However, this effect is absent from higher elasticity cases, though.

The transverse plots of the longitudinal velocity component at $x / H=0.6$ in Fig. 13(a) confirm that large levels of fluid elasticity, specifically the case $\mathrm{De}=1$, produce an intense change in the flow pattern, with velocity profiles tending to a strong, wide central jet with $u / U_{\text {in }} \approx 1.4$ for $\mathrm{De}=1.0$ and a near-wall layer of $0.30 \mathrm{H}$ thickness of slow fluid, not exceeding $u / U_{\text {in }} \approx 0.4$. For the lower Deborah number flows, similar effects are encountered in the shapes of Fig. 13(a), although to a lesser extent.

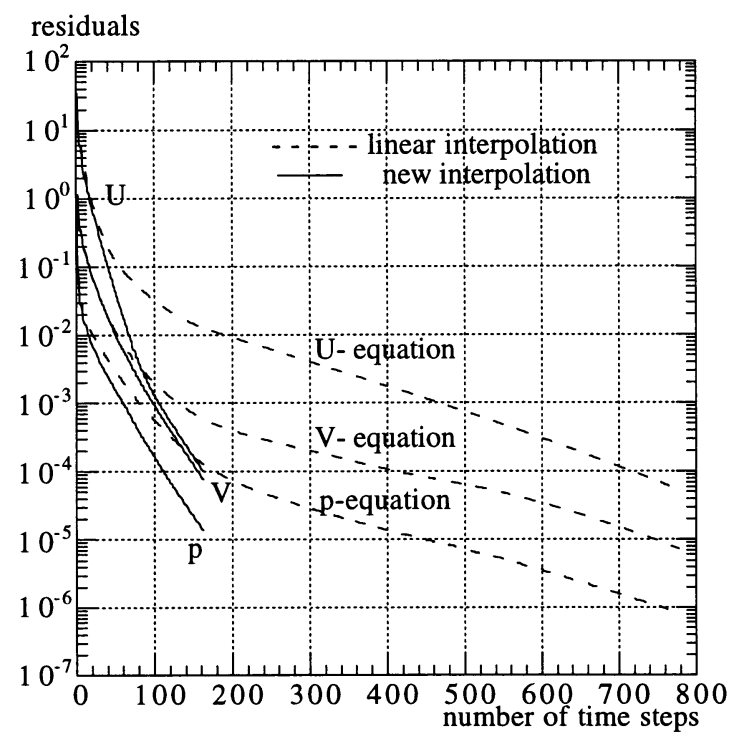

Fig. 11. Decay of the residuals with the linear and the new interpolation schemes for the stresses. 
a)

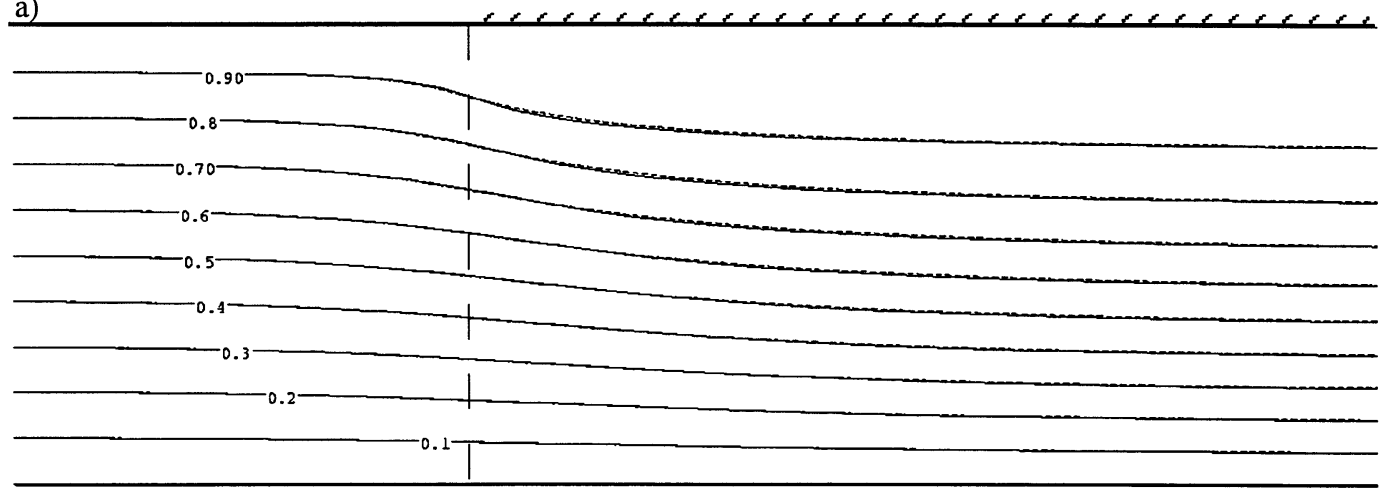

b)

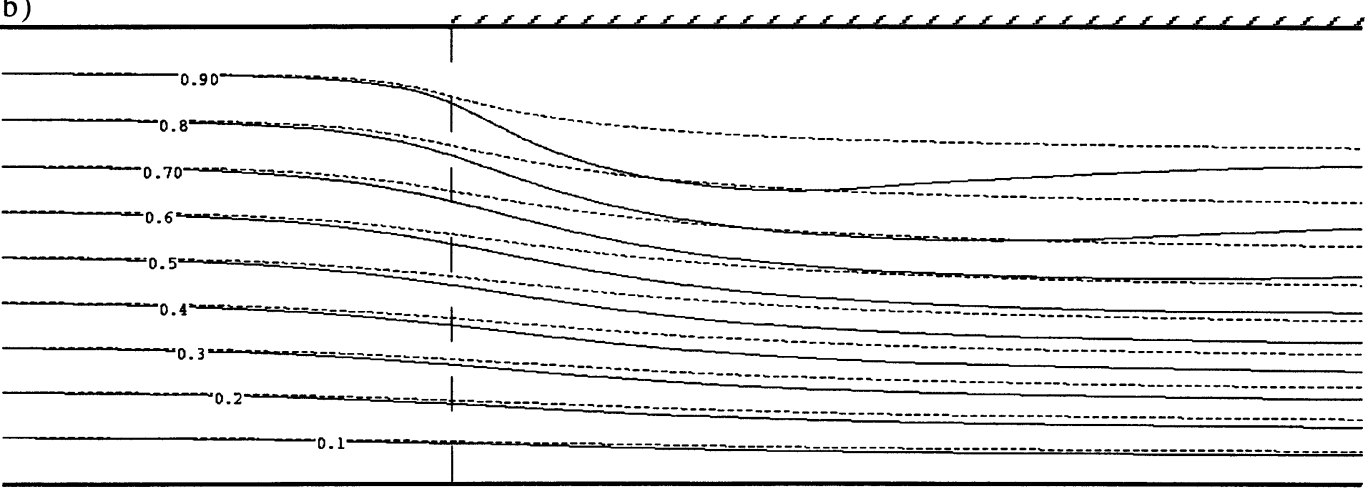

Fig. 12. Streamlines for $\mathrm{Re}=20$. (a) $\mathrm{De}=0$, LUDS (dashed line) and $\mathrm{De}=0.1$, LUDS (full line); (b) $\mathrm{De}=0.0$, LUDS (dashed line) and De $=1.0$, UDS (full line). (zoom in the region $x / H=-1-+2$ in mesh 7).

Flow redevelopment is accelerated for low levels of viscoelasticity, however it is delayed for highly elastic fluids ( $\mathrm{De} \geq 0.5$ ) because the strong deceleration of fluid near the wall reduces the transverse transfer of momentum, the mechanism for flow development. This is shown in the plot of Fig. 13(b) taken at $x / H=2.0$; the velocity profiles for the various Deborah number flows, except for the $\mathrm{De}=0.3$ case, do not follow the fully developed curve and some of them differ from the theoretical curve by a large amount. The faster flow recovery for $\mathrm{De}=0.3$ actually results from the fact that the velocity profile for that particular flow case follows the fully developed curve in the near-wall region closer than the other curves do (Fig. 13(a)), and thus, a lesser amount of momentum transfer is required for flow redevelopment.

An understanding of these variations is gained by the comparison between the Newtonian and viscoelastic stress fields, corresponding to the Deborah numbers of Fig. 12. Contours of the normalised shear stress $\left(T_{x y}\right)$, longitudinal normal stress $\left(T_{x x}\right)$, transverse normal stress $\left(T_{y y}\right)$ and the first normal stress difference $\left(N_{1}=T_{x x}-T_{y y}\right)$ predicted in the finest mesh, are presented in Figs. 14-17, respectively. Note that the mesh is concentrated near the computational wall and the slight oscillations seen in the figure contours close to the symmetry planes (at bottom of figures) are due to the coarser mesh there (Fig. 7). 
Careful inspection of those contours shows that an element of fluid is subjected to the stress fields schematically outlined in Fig. 18(a) (viscoelastic) and Fig. 18(b) (Newtonian) in the two regions I and II drawn in Fig. 6. In region I, components of the stress tensor for the Newtonian and the viscoelastic fluid ( $\mathrm{De}=0.1$ ) have identical signs and similar magnitudes, except very close to the wall leading edge where the non-Newtonian stresses reach much higher values. Stresses of similar magnitude occur earlier in the Newtonian flow than in the viscoelastic fluid flow due to the convective effect on the stresses, thus an element of Newtonian fluid is subject to lower stress gradients than the viscoelastic fluid. All of the components of the stress tensor and their gradients decelerate the flow in the symmetry plane region, at $y / H=1$, as the wall is approached: the building up of a negative shear stress (Fig. 14) brakes the fluid flow as is typical of a situation where there is a wall at rest; an increasingly negative longitudinal normal stress (Fig. 15) acts compressively thus helping also to retard the flow longitudinally; finally, there are increased levels of traction acting on the $y$-direction as the slip-stick junction is approached (increasing positive $T_{y y}$, Fig. 16), forcing the flow away from the symmetry plane, a result to be expected from continuity. It should also be emphasized that the similar qualitative contributions of the two normal stresses upon the flow translates into the summation of their absolute values to compute a growing compressive (negative) first normal stress difference, everywhere in region I (Fig. 17).

In region II, a different situation arises: very close to the wall the shear stress $\left(T_{x y}\right)$ and the transverse normal stress $\left(T_{y y}\right)$ retain their directions, however, they are intensified, especially for the viscoelastic fluid. Subsequently, on moving downstream from the wall leading edge, the magnitudes of those two stresses quickly drop to similar levels, however, a very different
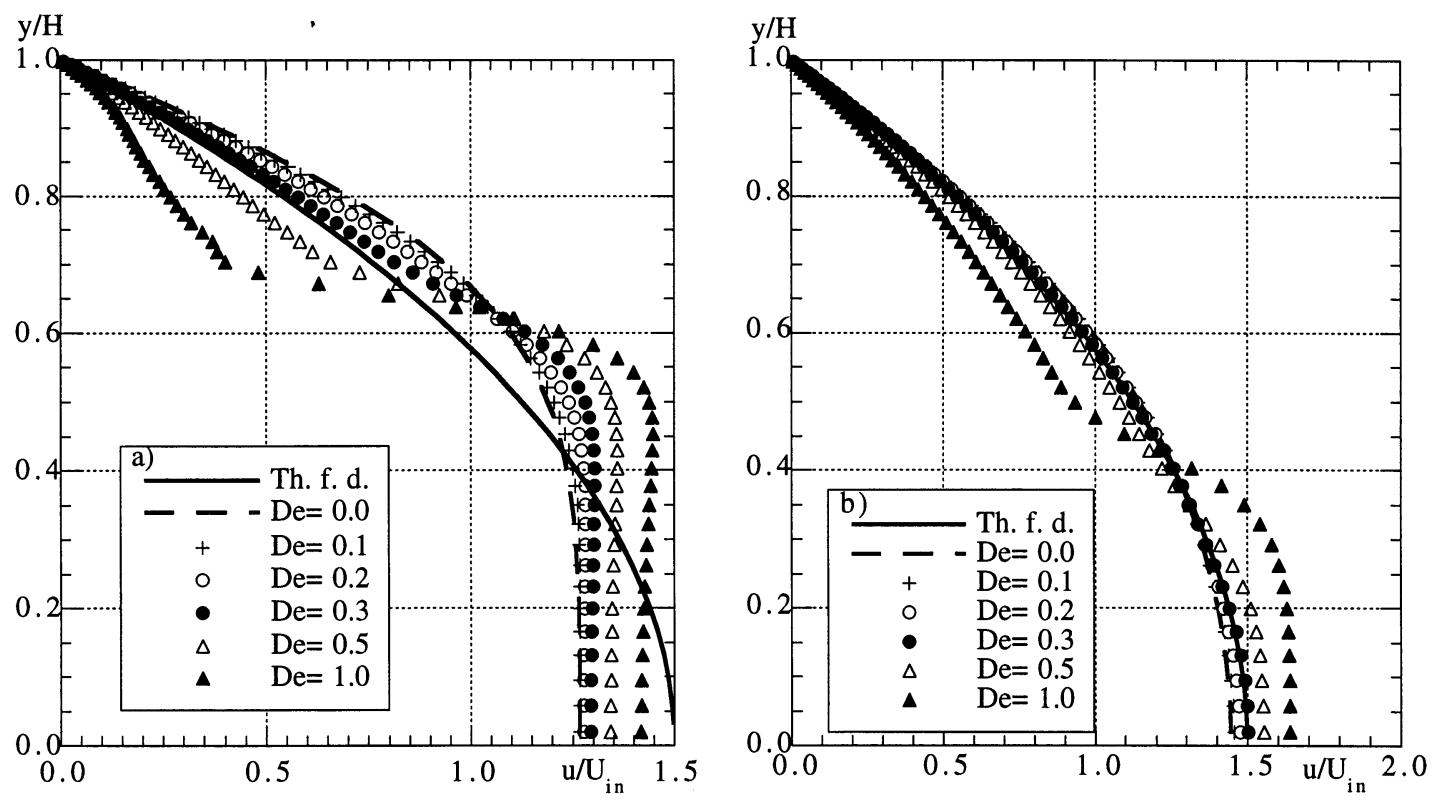

Fig. 13. Transverse profiles of the normalised longitudinal velocity for various Deborah number flows. (a) $x / H=0.6$; (b) $x / H=2.0$. ('Th. f. d.' refers to the theoretical fully developed axial velocity profile). 


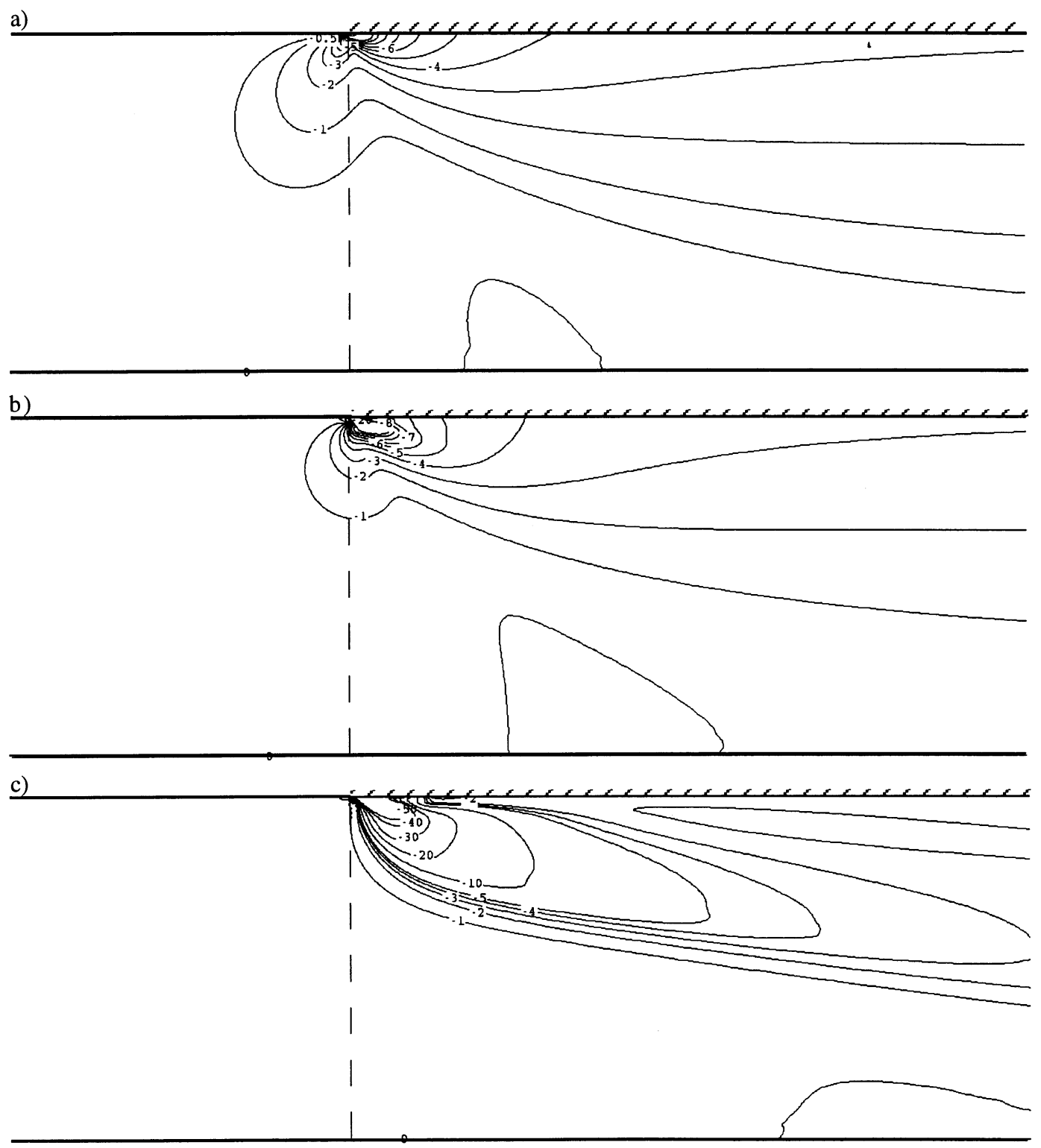

Fig. 14. Contours of $T_{x y}$ for a Reynolds number of 20: (a) De =0, LUDS; (b) De =0.1, LUDS; and (c) De =1.0, UDS.

behaviour is observed with the longitudinal normal stress $\left(T_{x x}\right)$. For the Newtonian fluid, this stress component is compressive (negative) and its variation and magnitude are much like those of the other stress components. However, for the viscoelastic fluid, $T_{x x}$ quickly increases to high positive values (traction), which are one order of magnitude higher than those for the Newtonian fluid, and later decreasing to values still positive, thus always defining a stress gradient which is opposite in sign to that of Newtonian fluids. The contours of $T_{x y}$ and $T_{x x}$ for 
$\mathrm{De}=1$ viscoelastic flow show clearly a stress boundary-layer with high stress values, and its boundary emerging from the wall discontinuity being convected downstream.

In the wall region II of Fig. 6, both $T_{x x}$ and $T_{y y}$, and equally their variations, act to decelerate the flow of the Newtonian fluid, i.e. they both contribute negatively to the first normal stress difference, whereas an element of Non-Newtonian fluid initially suffers a strong traction in the longitudinal direction, forcing the alignment of the streamlines with the main direction of the
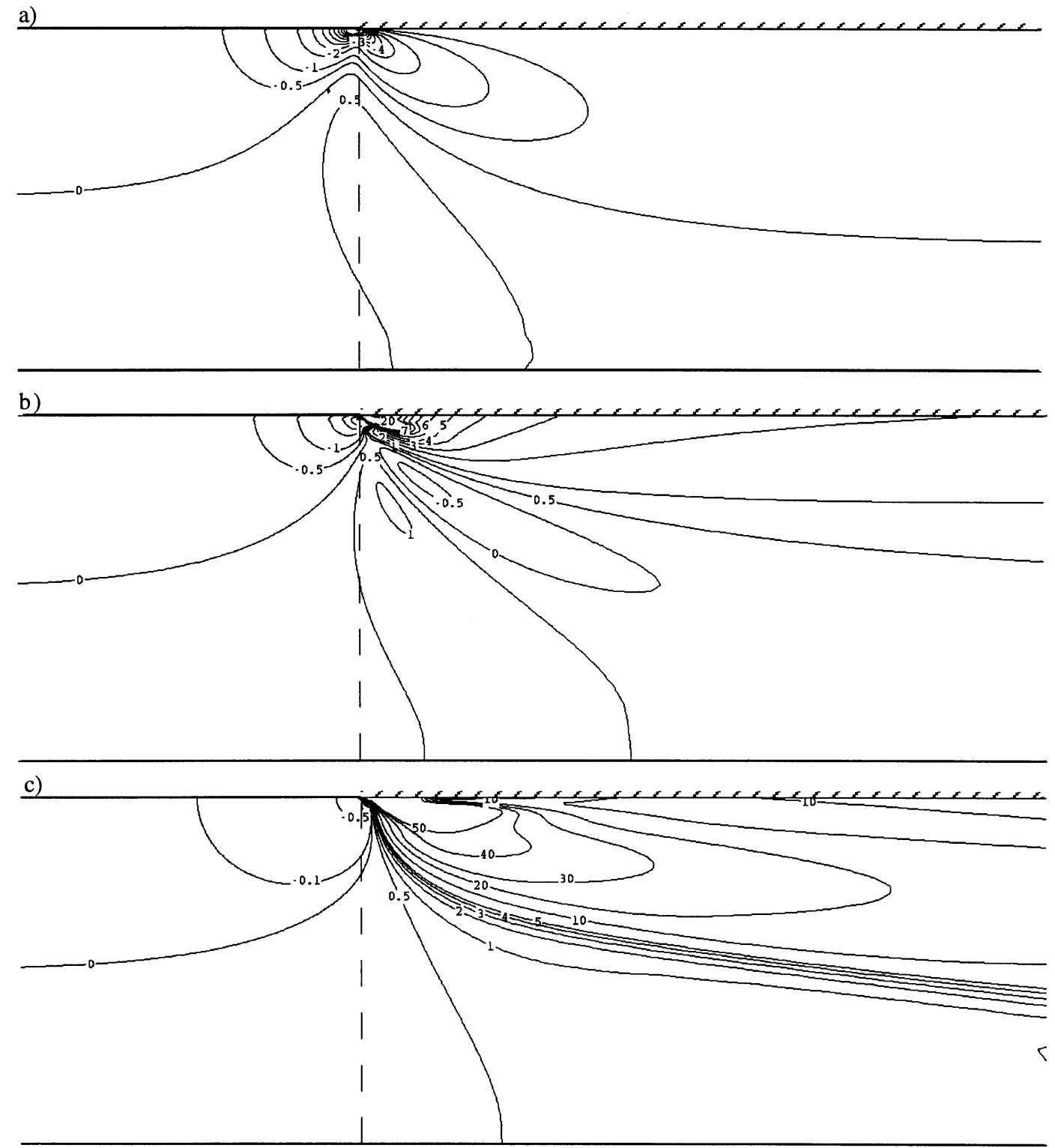

Fig. 15. Contours of $T_{x x}$ for a Reynolds number of 20: (a) De =0, LUDS; (b) De =0.1, LUDS; and (c) De =1.0, UDS. 


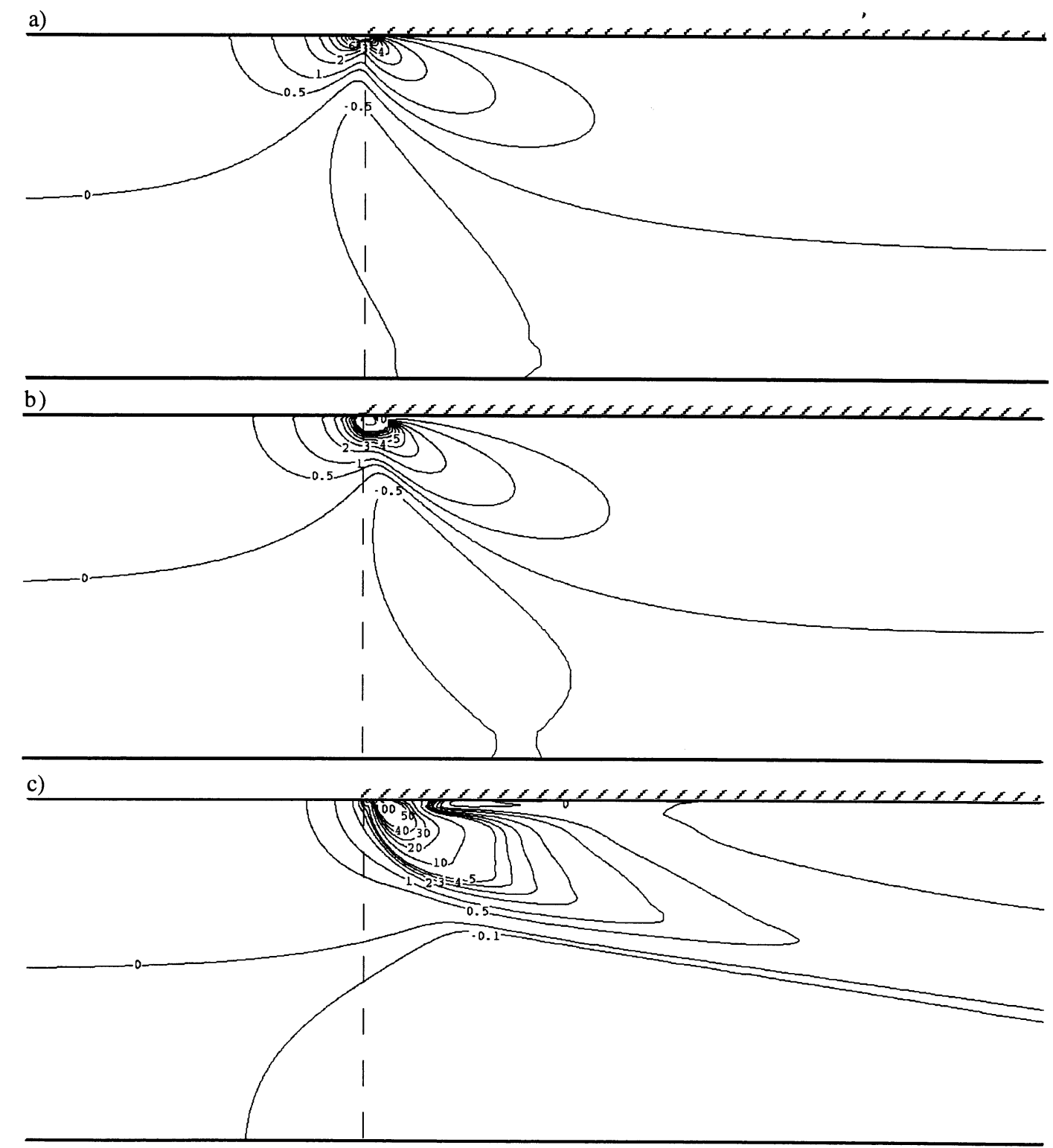

Fig. 16. Contours of $T_{y y}$ for a Reynolds number of 20: (a) De =0, LUDS; (b) De =0.1, LUDS; and (c) De $=1.0$, UDS.

flow, and later the traction is released and the flow acquires lateral velocity and converges towards the wall. Figs. 15 and 16 show that for the $\mathrm{De}=0.1$ flow, $T_{x x}$ is much larger than $T_{y y}$ in region II, as confirmed in the first normal stress difference plot of Fig. 17. Therefore, in spite of the tensile transverse normal stress acting to create a negative lateral velocity, the predominant effect is that of the initial intense increase and later relaxation of positive $T_{x x}$. As a result, the Non-Newtonian flow eventually acquires a positive lateral velocity and moves towards the 
wall (see streamline plots of Fig. 12). Note that the action of the stress field upon the hydrodynamics is carried out via its gradients, rather than through the stresses themselves.

Within the decelerated near-wall region for the viscoelastic fluid flow, the shear rates are low, and thus the transverse transfer of momentum by molecular diffusion is reduced in comparison to the Newtonian fluid flow. Therefore, it is not surprising to conclude from Fig. 13 that flow redevelopment is delayed for strong levels of viscoelasticity.
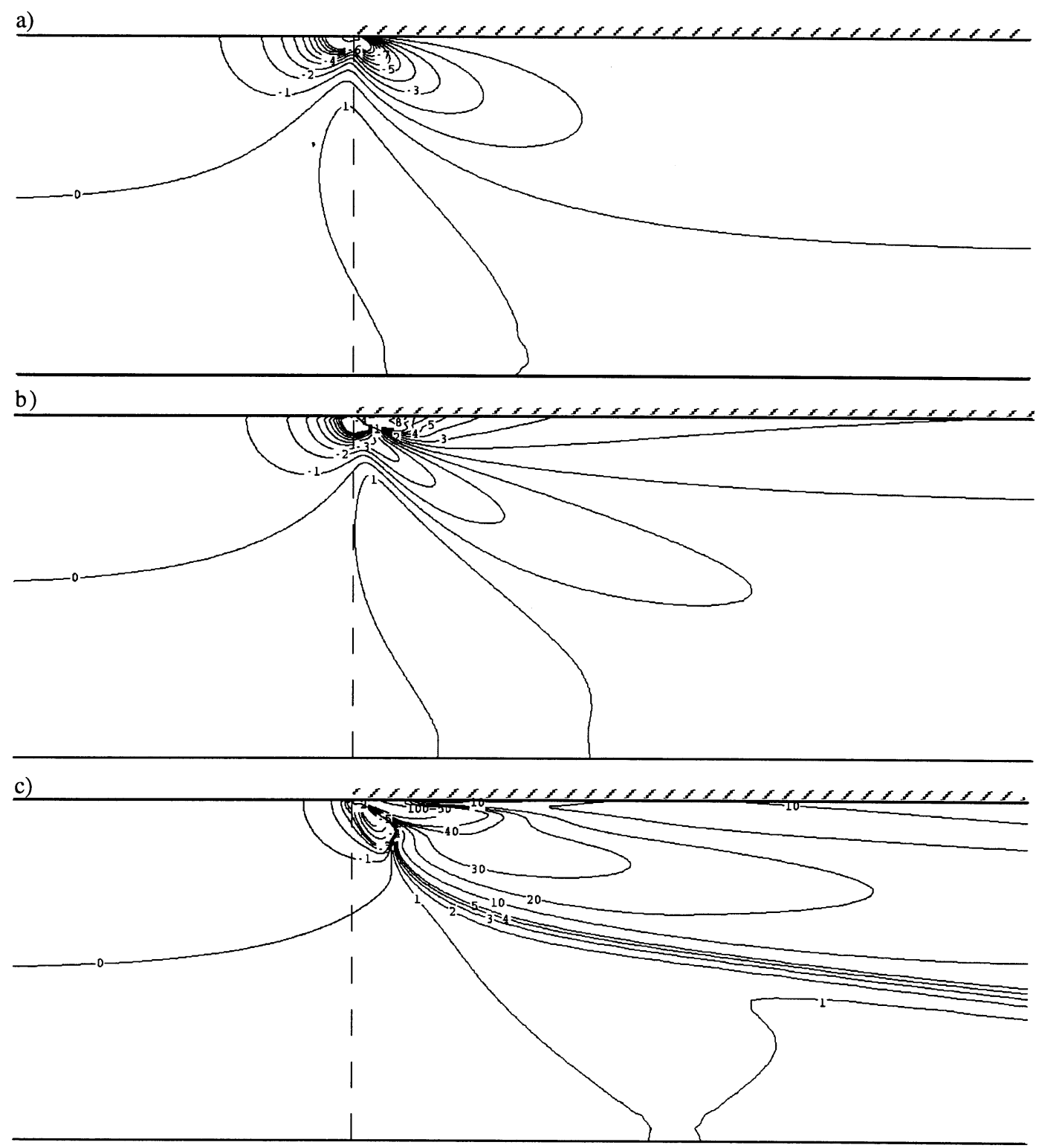

Fig. 17. Contours of $T_{x x}-T_{y y}$ for a Reynolds number of 20: (a) De=0, LUDS; (b) De =0.1, LUDS; and (c) $\mathrm{De}=1.0$, UDS. 


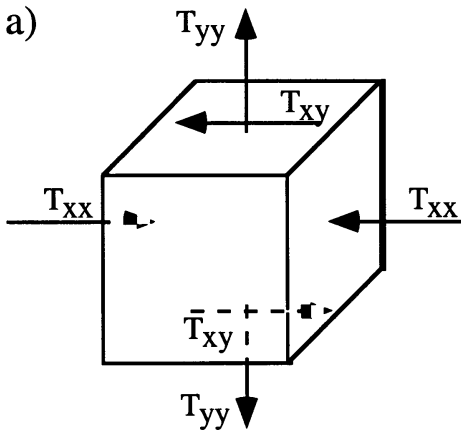

Region I

b)

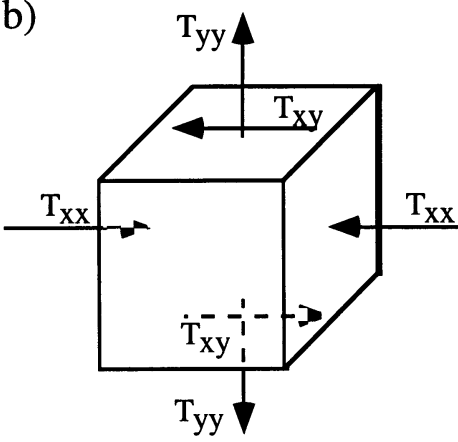

Region I

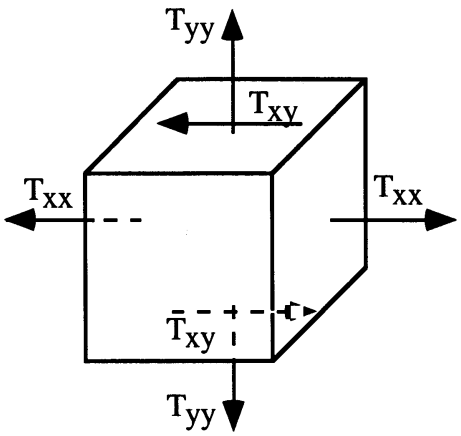

Region II

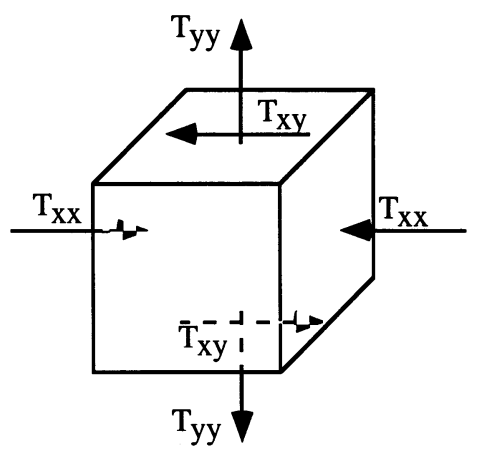

Region II

Fig. 18. Stress field acting upon an element of fluid in regions I and II of Fig. 6: (a) Viscoelastic fluid (De $=0.1$ ); (b) Newtonian fluid $(\mathrm{De}=0)$.

In the comparison between the two lower Deborah number flow cases ( $\mathrm{De}=0$ and 0.1 , respectively), the mean flow pattern does not differ significantly (Fig. 12(a)). As a result, the differences in the stress field and its gradients can be attributed mainly to the new term of the constitutive equation rather than to differences in the term $2 \eta \boldsymbol{D}$. This is also readily seen by realizing that, due to continuity, $T_{x x}=-T_{y y}$ for the Newtonian fluid. From Fig. 16, it is seen that $T_{y y}$ does not differ significantly for the $\mathrm{De}=0$ and 0.1 cases, however, in zone II, $T_{x x}$ for $\mathrm{De}=0.1$ is completely different from $T_{x x}$ for $\mathrm{De}=0$ (Fig. 15). Thus, these differences are to be explained by the elastic term in the constitutive equation and its stronger action via the $T_{x x}$ component.

For increased viscoelasticity $(\mathrm{De}=1.0)$, differences in flow pattern are enhanced and though the viscous term $2 \eta \boldsymbol{D}$ may now also partly contribute to the differences seen in the stress field, the higher value of the time constant $\lambda$ leads to a much stronger effect of the elastic convective-like terms in the constitutive equation. Note that the pattern of the contours of all stress tensor components for $\mathrm{De}=1.0$ is very different to those of $\mathrm{De}=0.1$ and 0.0 , with the former strongly suggesting a convective effect on the downstream transport of stresses (see Fig. 14(c) where the convected $T_{x y}$ pattern contributes to the decelerated flow in the near-wall region of Fig. 13(a)). 


\subsection{Entry flow: stress discontinuity}

The sudden change in boundary condition leads to very high values of the predicted stresses (theoretically tending to infinity, Fig. 8) akin to the singular behaviour reported in the literature concerning the salient corner in a sudden contraction and the stick-slip problem [2,10,21]. The predicted variations of all stress components along the line $y / H=0.985$ (close to the wall) are smooth, as seen in Fig. 8 and similar variations at $y / H=0.995$ (the line almost along the wall, passing through the singular point) not shown here, are also free from oscillations and the only remarkable feature is the very high peak of $T_{x x}$ attaining a value of $\sim 250$.

Predicted transversal profiles of the axial velocity component at several stations just upstream of the wall leading edge (Fig. 19), and of the lateral velocity component at stations downstream of that point (Fig. 20), illustrate the quality of the present results. In fact, the gradual decay of ' $u$ ' as the fluid senses the approaching wall is predicted very smoothly (Fig. 19) as compared with similar profiles presented by [2], where large oscillations are presented. These authors mention that the localised oscillations in the region around the singular point do not affect the quality of the results at some distance from that point (as reflected in the profiles in Fig. 9), and the same sort of behaviour was found in this work. At an early stage of the development of the method, analytical expressions for the stresses at a wall have been used instead of Eq. (61), namely $\tau_{x y}=\eta \partial u / \partial y$ and $\tau_{y y}=2 \lambda \eta(\partial u / \partial y)^{2}$ for a wall at constant $y$. These analytical expressions are not consistent with the numerical approximations used at the cells adjacent to a wall plane and result in oscillations in the predicted profiles, akin to those of [2], but which did not affect the results at some distance from the wall. It is now clear from the results in Figs. 19-22) that those former oscillations were due to the treatment of the boundary conditions and that they are effectively removed by using Eq. (61) as the solid wall condition.

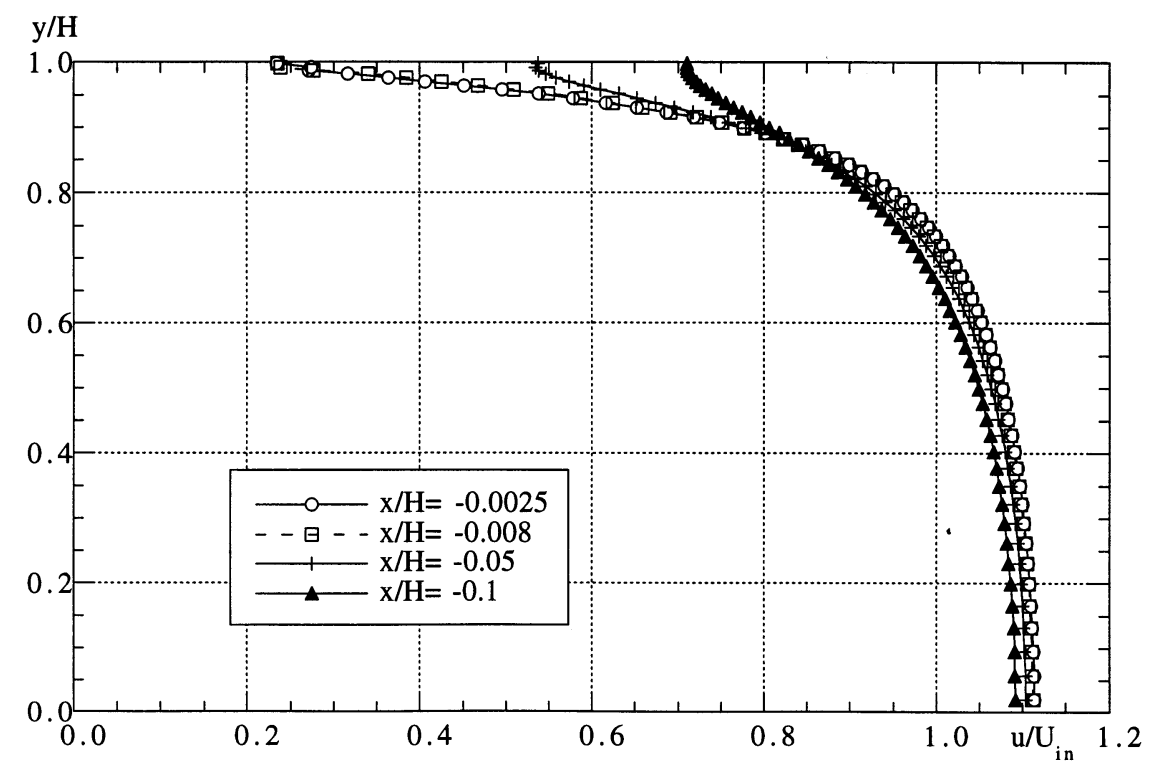

Fig. 19. Transverse profile of the normalised longitudinal velocity component just upstream of the slip-stick junction for $\mathrm{De}=0.1, \mathrm{Re}=20$ and LUDS. 


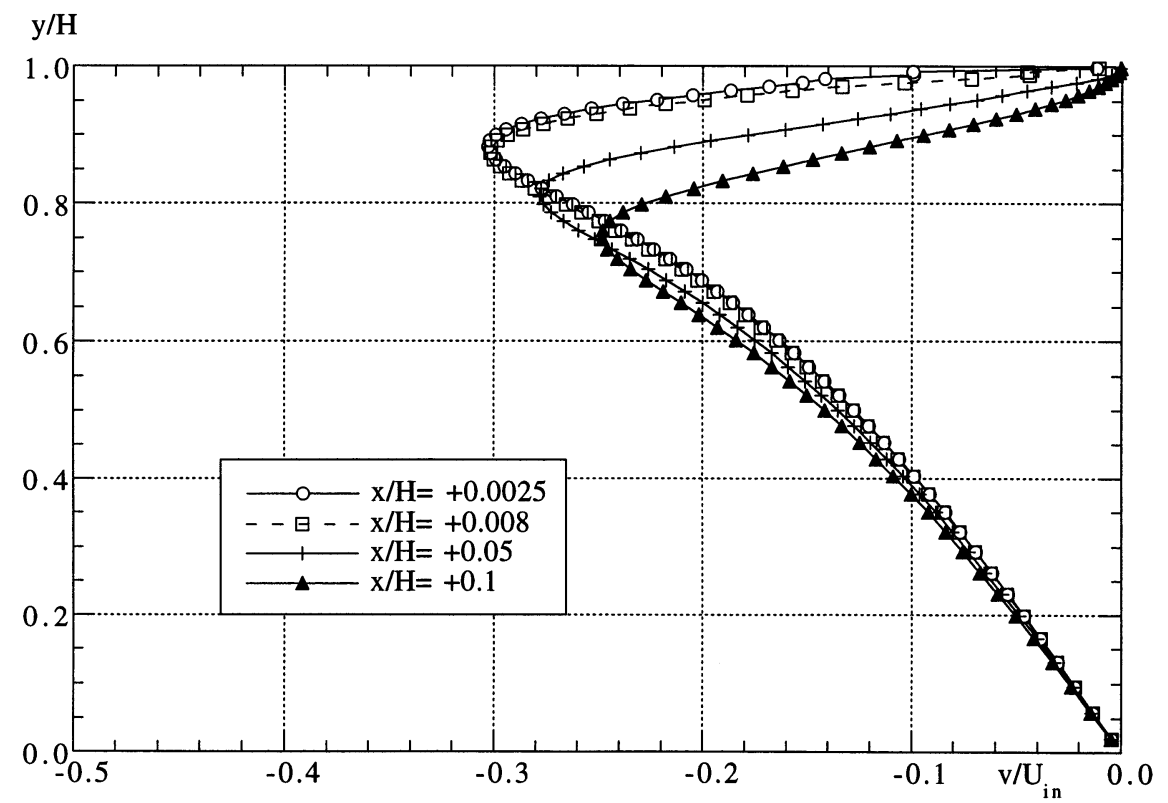

Fig. 20. Transverse profile of the normalised transverse velocity component just downstream of the slip-stick junction for $\mathrm{De}=0.1, \mathrm{Re}=20$ and LUDS.

Very sharp stress boundary-layers develop from the wall leading edge at $x / H=0$ as seen in the few transverse profiles of the shear and normal stress in Figs. 21 and 22 and in the contour plots (Figs. 14-16). In Fig. 21(a), the shear stress must be zero at $y / H=1$, the symmetry line. However, since the fluid senses the approaching wall, $T_{x y}$ builds up in the layer immediately below the $y / H=1$ line. At the leading edge, $T_{x y}$ jumps from zero to a very large value (order 100 , theoretically it would be infinity) and then the flow adjusts itself to the presence of the wall, $T_{x y}$ falls to values appropriate to those due to viscous wall friction, and this reduction is accomplished in the relative short distance of $x / H \approx 0.1$. The axial normal stress $T_{x x}$ follows a similar variation (Fig. 22(a-b). It is interesting to note that the maximum values of both $T_{x y}$ and $T_{x x}$ at $x / H \approx 0.1$ are located at some distance below the wall, a feature certainly associated with the convective and the convective-like terms in the constitutive equations which act to transport the maxima of $T_{x y}$ and $T_{x x}$ seen just upstream (Fig. 21(a) and Fig. 22(a)) to the line below $y / H=1$. This feature is also seen in the contours of $T_{x y}$ and $N_{1}$ presented in Fig. 14(b) and Fig. 17(b), respectively; it is accentuated for higher elasticity (De =1, Fig. 14(c) and Fig. 17(c)).

\subsection{Entry flow: non-orthogonal mesh}

The entry flow at a Deborah number of 0.1 and a Reynolds number of 20 was also computed with the non-orthogonal mesh 7 of Fig. 7 and the LUDS interpolation scheme. The results were virtually the same as for the orthogonal mesh reported above and Fig. 23 shows the corresponding contour map of $N_{1}$. As expected, the figure coincides with the corresponding contour plot of Fig. 15(b) obtained with the orthogonal mesh. 
It remains to be seen whether the algorithm performs equally well in intrinsically non-orthogonal geometries, the subject of the next section.

\subsection{Flow around a confined and unbounded cylinder}

In this section, some predictions are presented for the confined and unbounded flow of an UCM fluid past a circular cylinder. In the confined case, the base blockage ratio is $\beta \equiv a / H=$ 0.5 , where $a$ is the cylinder radius and $H$ is the half width of the channel. This particular case has been put forward as a new bench-mark problem during the 8th Workshop on Numerical Methods [23] with the justification that it is more amenable to birefringence measurements of the stress field than the related case of the sphere in tube flow. There are not many works for this particular geometry, however, some recently published finite-element results [20] are sufficient for the present validation. The main concern here is to demonstrate that the non-orthogonal semi-structured capability of the present finite-volume procedure is capable of coping with this non-rectangular geometry, both for the Newtonian and viscoelastic fluid cases.

The flow geometry can be inferred from the zoomed portion of the mesh shown in Fig. 24(a). A streamwise uniform velocity $U$ enters the domain at $x=-20 a$ and leaves at $x=60 a, x=0$ being the position of the cylinder axis. Both the channel wall, at $y=H=2 a$, and the cylinder surface are considered as non-slip surfaces, and the centre-line at $y=0$ is a symmetry plane. The channel wall moves with the same uniform velocity $U$. The mesh was generated with six mesh-generating blocks (one inlet portion, one outlet portion and four blocks around the cylinder, at $45^{\circ}$ angles) within which a mesh was constructed with quadratic isoparametric functions. Since this problem serves as an illustration of the capabilities of the present finite-volume method, no systematic mesh refinement study has been undertaken as in the
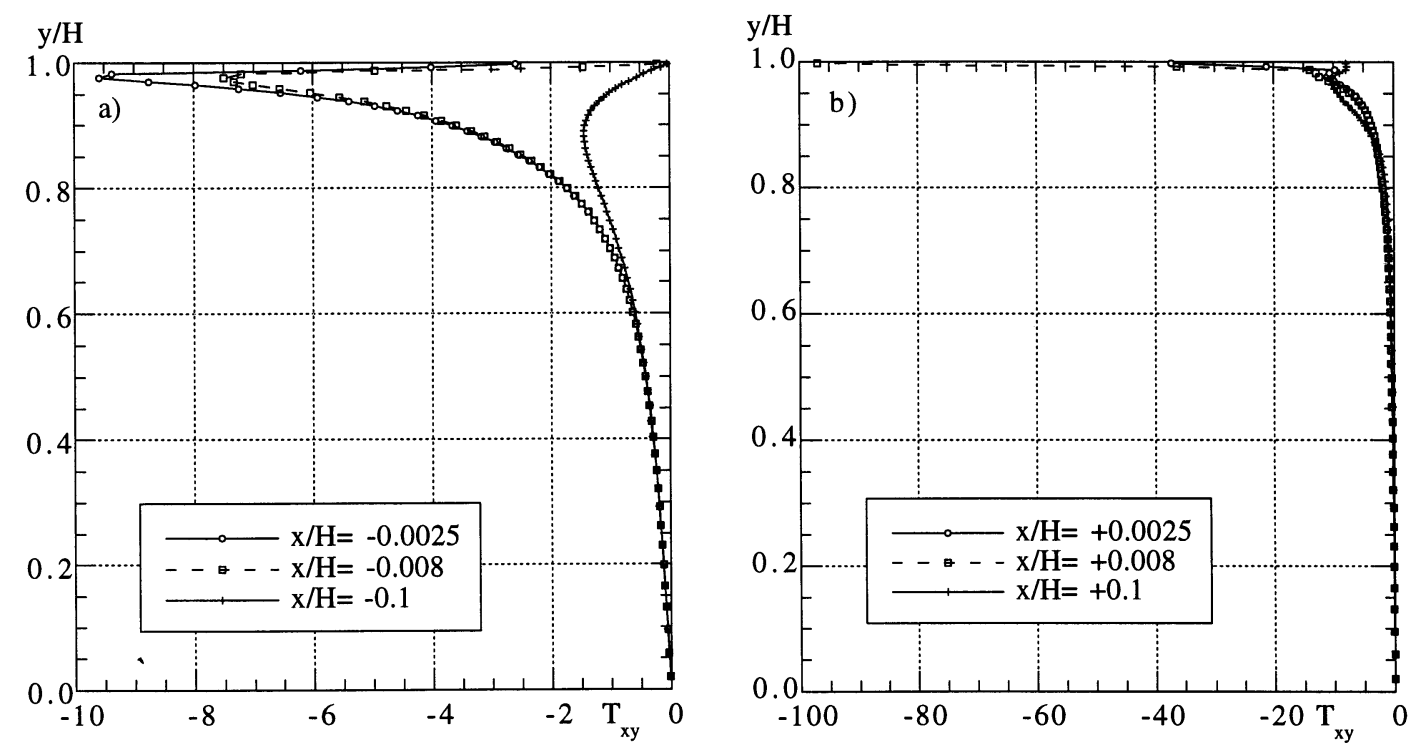

Fig. 21. Transverse profiles of the normalised shear stress in the near vicinity of the slip-stick for $\mathrm{De}=0.1, \mathrm{Re}=20$ and LUDS: (a) upstream profiles; (b) downstream profiles. 

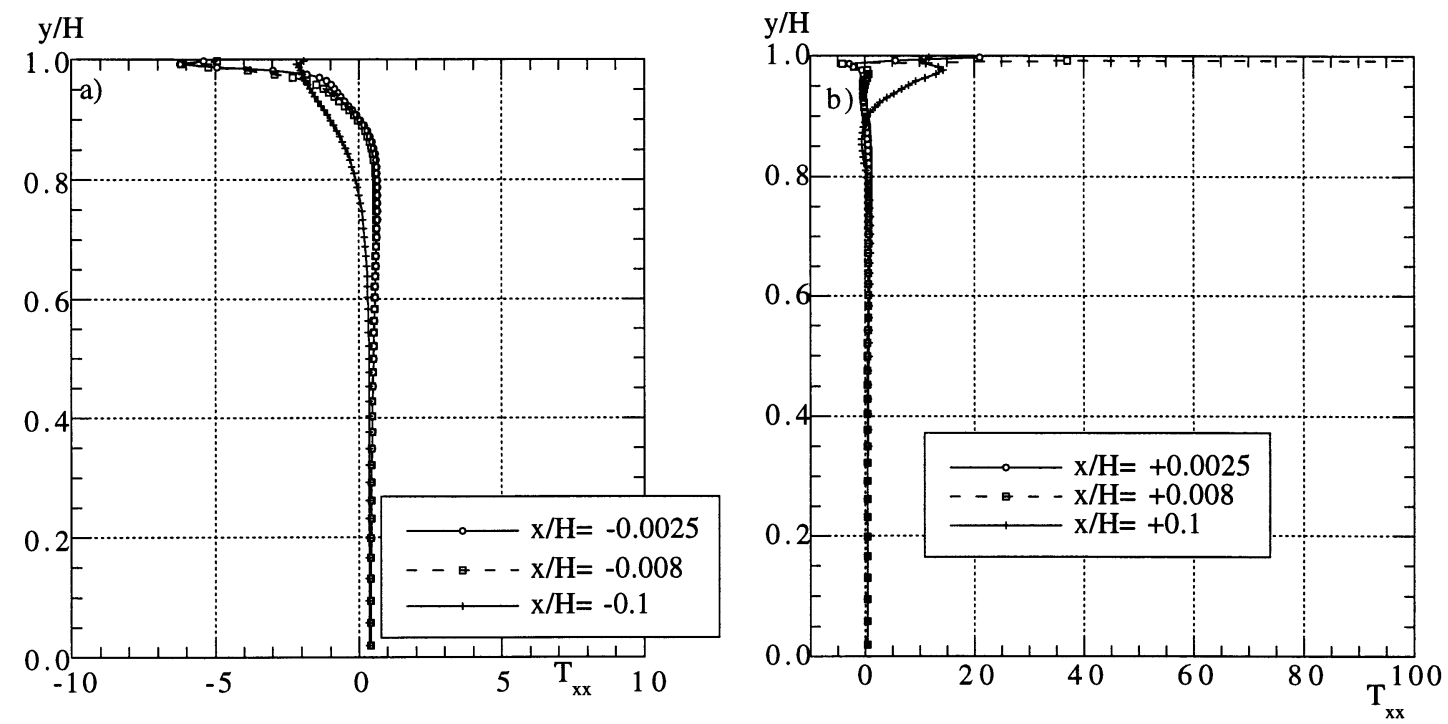

Fig. 22. Transverse profiles of the normalised longitudinal normal stress in the vicinity of the slip-stick for De $=0.1$, $\mathrm{Re}=20$ and LUDS: (a) upstream profiles; (b) downstream profiles.

previous entry flow problem. The calculations have however been performed with two meshes, one having 3000 control-volumes with the smallest spacing of $\delta x=\delta y=0.05 a$ (mesh 1), and the other $\delta x=\delta y=0.025 a$ (mesh 2) having 12000 control-volumes. The mesh fineness of mesh 1 is of the same magnitude as that in [24] for the related sphere-in-tube problem (they have 15852 unknowns with the $\tau_{\theta \theta}$ stress, not required here, also accounted for, and we have 18000 unknowns). Although these authors have considered the sphere-in-tube problem, it is emphasised that the two cases have similar flow patterns and, in a numerical point of view, the resolving capacity of meshes for each case are directly comparable. An assessment of the accuracy of the results can also be made from a comparison using the UDS and the LUDS schemes as will be shown.

Fig. $24(\mathrm{a}-\mathrm{d})$ show the mesh, the contours of the normalised first normal stress-difference of the normalised shear stress (always referred to the Cartesian axis) and the streamlines, respectively, for the case $\operatorname{De} \equiv \lambda U / a=1$ and $\operatorname{Re} \equiv \rho U 2 a / \eta=1$ using the LUDS scheme. The maximum and minimum normalised values of the stresses are 74.5 and -60.6 for $N_{1}$ and 35.1 and -22.4 for $T_{x y}$, respectively, compared with 13.1 and -13.7 for $N_{1}$ and 10.9 and -7.1 for $T_{x y}$ for the Newtonian case; these values occur at the cylinder surface, where strong compression is observed in front of the cylinder and at an angle of about $125^{\circ}$, when the flow is subject to high shear rates due to the flow acceleration and blockage. There is also a local maximum of the first normal stress difference downstream of the rear stagnation point, in the wake, a feature similar to that observed by Bush [25] in the wake of a sphere. According to this author, the maximum normal stress in the wake for the sphere flow was associated with the high strain rates found there, which are intensified by the presence of the pipe walls. A similar feature is bound to occur here, although to a lesser extent, since in the cylinder case the flow is locally closer to a planar extension rather than to the stronger pure elongational flow occurring behind the sphere. 
These features of the first normal stress difference are reflected on the downstream shift of the streamlines, as compared with the $\mathrm{De}=0$ case (Fig. 24(d)). It is as if the fluid stored elastic energy while submitted to compression in front of the cylinder, followed by elastic relaxation after the fluid has passed the cylinder, thus inducing the bulging in the streamlines seen in the figure. Manero and Mena [26] have observed the downstream shift of the streamlines for De $\leq 1$ flows and an upstream shift for higher Deborah number flows. However, there is no consensus in the literature on exactly by what conditions the streamlines tend to shift upstream [20]. The usual result is a downstream shift, as reflected in the reduction of the drag coefficient with the Deborah number presented by most authors, except in a very localised region close to the cylinder rear stagnation point, where a fluid acceleration higher than in the Newtonian case is observed [25]. This feature is also observed in the streamlines of Fig. 24(d).

For a quantitative comparison with the simulations of [20], results of the drag coefficient $\left(C_{\mathrm{D}}\right)$ are plotted in Fig. 25 as a function of the Deborah number. The drag coefficient is defined as the force on the cylinder per unit length divided by $\left(0.5 \rho U^{2} a\right)$ and the three points in Fig. 25 are from Huang and Feng for $\beta=0.5$ and $\mathrm{Re}=1$ and with an Oldroyd-B fluid $\left(\lambda_{2} / \lambda_{1}=0.125\right)$. The agreement is good for the Newtonian flow case $(\mathrm{De}=0)$ and moderately good for the other cases, $(\mathrm{De}=0.1$ and 1$)$, which are predicted by Huang and Feng to be slightly higher and lower than the present predictions, respectively. The figure also allows for an interesting comparison between the results obtained with the UDS and the higher-order LUDS interpolation schemes together with the effect of mesh refinement: for mesh 1, the results of the two schemes agree up to a Deborah number of 0.7 , resulting in the drag coefficient predicted by UDS levelling out and starting to increase, while LUDS predicts a systematically decreasing variation. The figure also

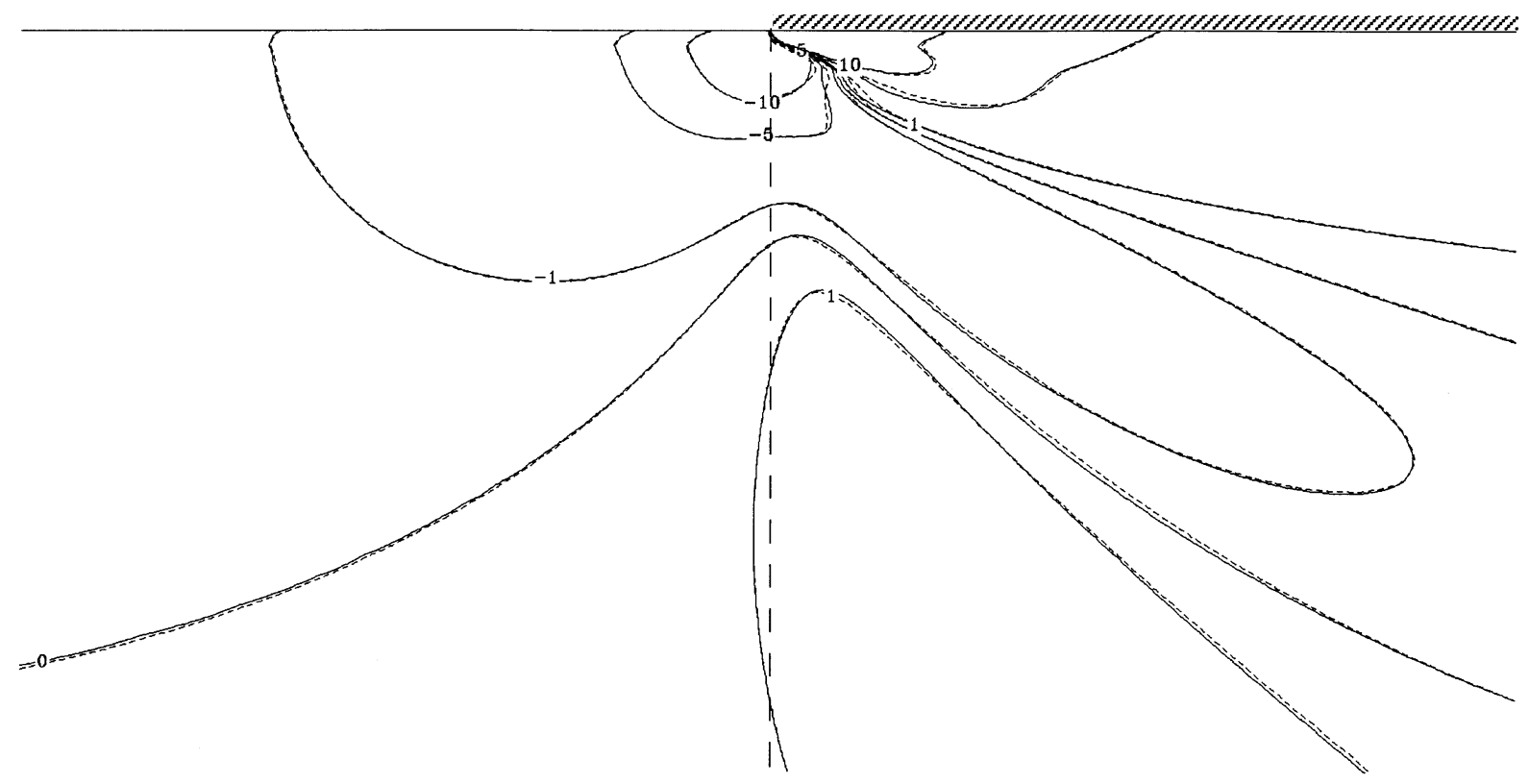

Fig. 23. Zoomed view of the $N_{1}$ contours around the singularity $(x / H=-0.05-+0.05$ and $y / H=+0.05-1)$, for $\mathrm{Re}=20$, De $=0.1$, LUDS. Full line (non-orthogonal mesh); broken line (orthogonal mesh) 

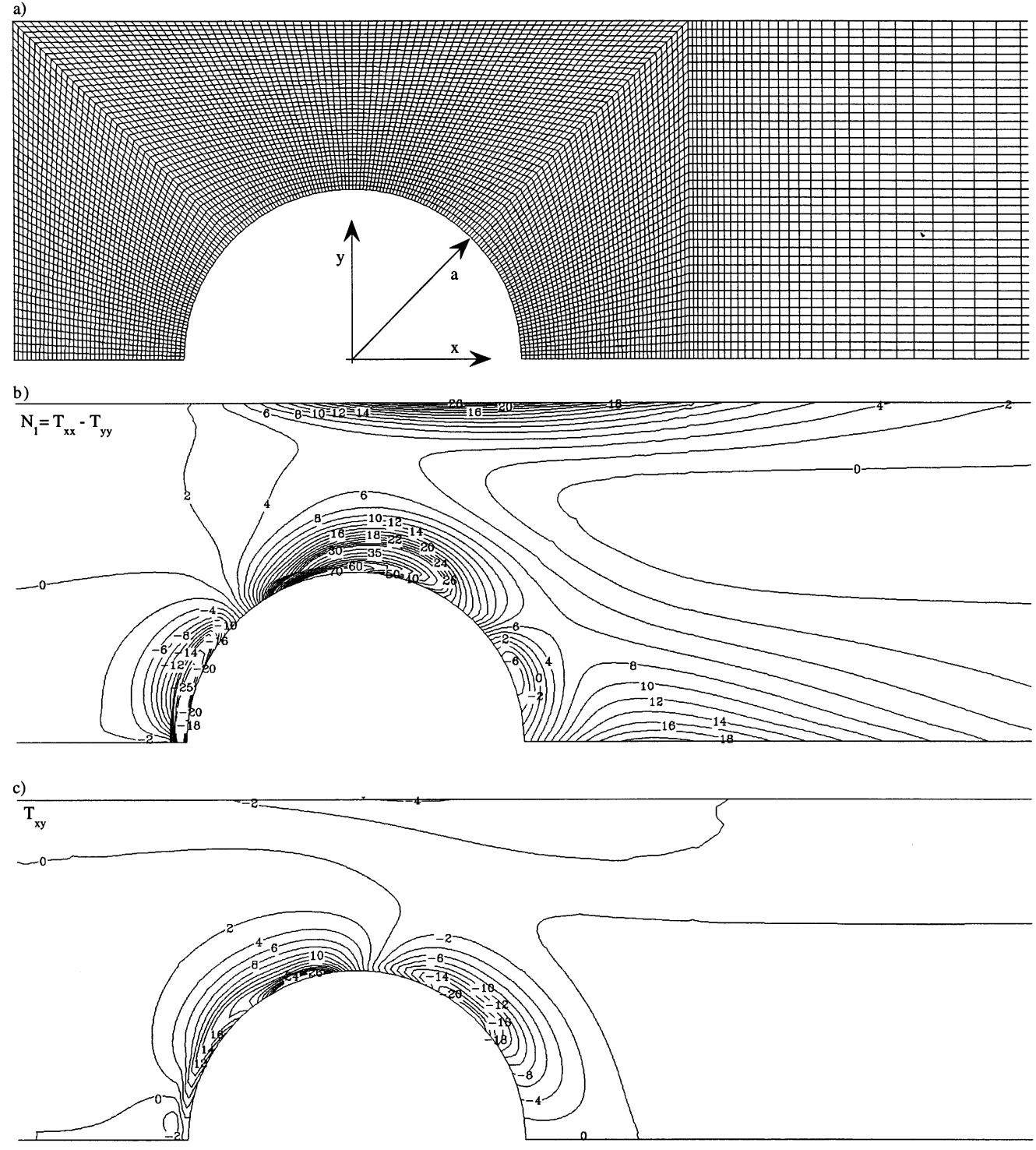

d)

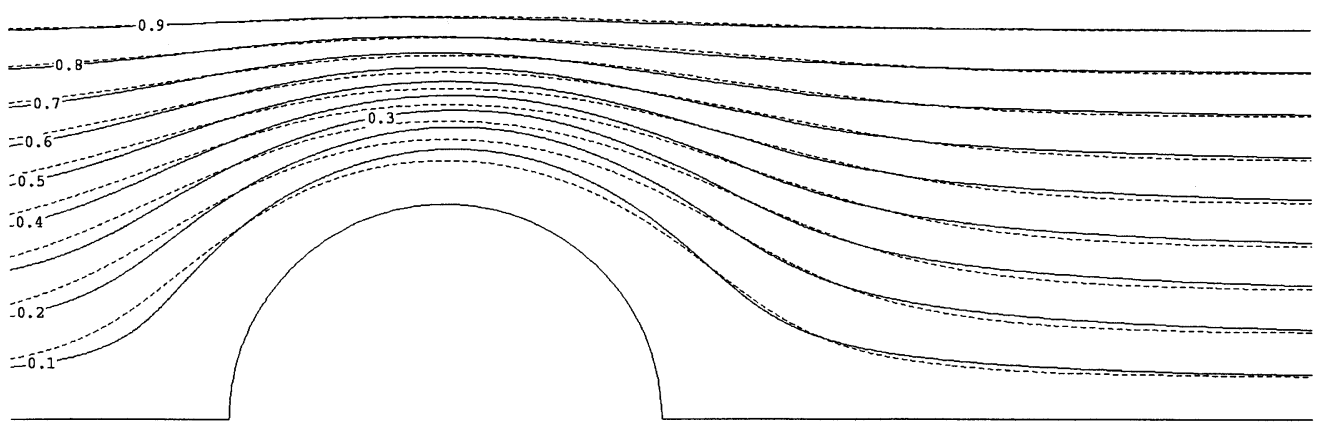

Fig. 24. Flow around a bounded cylinder for $\operatorname{Re}=1, \mathrm{De}=1, \beta=0.5$ and LUDS: (a) Mesh 2; (b) Normalised $N_{1}$; (c) $T_{x y}$; (d) Streamlines (solid lines $\mathrm{De}=1$, broken lines $\mathrm{De}=0$ ). 


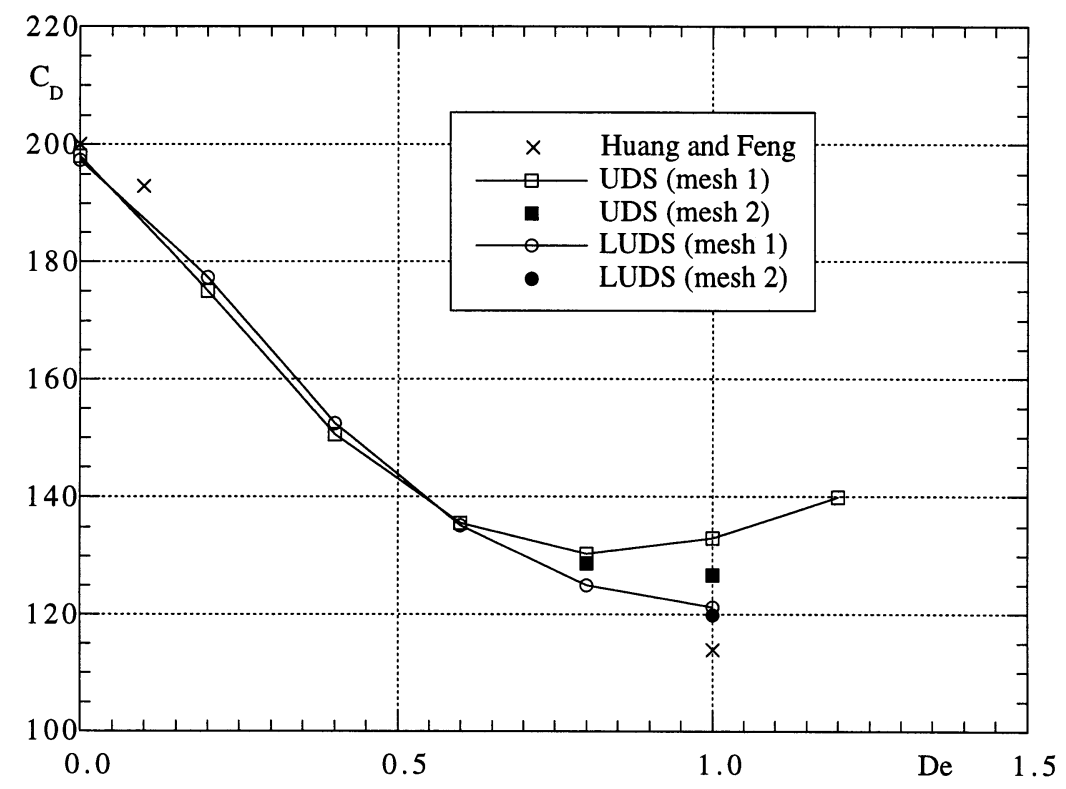

Fig. 25. Drag coefficient on a circular cylinder with a blockage ratio of 0.5 , as a function of the Deborah number. Effect of the interpolation method.

suggests that further mesh refinement is still required if UDS is to be used for De $\geq 0.7$, whereas results with LUDS are expected to be more accurate and the resolution provided by mesh 1 may be sufficient. This is confirmed from the results of the calculations performed with the finer mesh 2 with both schemes (closed marks in Fig. 25). The amount of numerical diffusion produced by the UDS calculations in the constitutive equations was much reduced with mesh refinement and the results approached those obtained with the second order LUDS scheme, while the LUDS results were barely affected by mesh refinement $\left(C_{D}\right.$ differs by only $1.1 \%$ for $\left.\mathrm{De}=1\right)$.

A similar situation can be found in the literature for the sphere-in-tube problem [27] for which earlier results also show a minimum in the drag coefficient versus Deborah number curve. However, more recent and accurate predictions tend to show that $C_{\mathrm{D}}$ levels out and does not increase with De (at least for the range of De in which a solution can be obtained).

The velocity variation along the centerline, within the cylinder wake, is compared with results from Huang and Feng [20] in Fig. 26 for the Deborah number flows of 0 and 1, at a constant Reynolds number of 1 and blockage ratios of $\beta=0.5$ (Fig. 26(a)) and $\beta=0.33$ (Fig. 26(b)). Again, the Newtonian cases are in perfect agreement, lending some support to the correctness of the non-orthogonal capability of the finite-volume method. The viscoelastic case with $\beta=0.33$ also shows very good agreement, whereas the higher blockage case shows an opposing behaviour: Huang and Feng predicted a shorter wake with some velocity overshoot, whereas we predict a larger wake than for the Newtonian case with a very slight overshoot much further downstream. This opposing trend is related to the fact that we predict a downstream shift of the streamlines (Fig. 24(d)) whereas Huang and Feng mention an upstream streamline shift. Huang and Feng discuss this problem and do mention that the UCM fluid tends to behave differently than the Oldroyd-B fluid. This may be the case for the disagreement for the $\mathrm{De}=1$ flow case in 
Fig. 26(a), however, it is necessary that this question be further investigated. It may be added that the shape of the predicted velocity profiles in the wake follows the same trends with an increasing Deborah number as in the measurements of Bush [25] in contrast to Huang and Feng.

In his investigation of the shift of streamlines in the sphere flow, Bush [25] has also stressed the enormous influence of the fluid rheology upon this flow characteristic, especially whenever there are regions of intense elongational flow. The reason for this can be the following: the presence of walls increases the shear rates on the cylinder surface closest to the wall and enhances the elongational character of the flow downstream of rear stagnation point. Since the retardation time term of the Oldroyd-B model affects the first normal stress difference and the elongational viscosity [28], the reduction of the wall effect decreases the influence of that term and improves the comparison between predictions with the UCM and Oldroyd-B models [29].

Due to the discrepancy with Huang and Feng for the $\beta=0.5$ case and in order to better assess predictions with viscoelastic fluids in this non-orthogonal flow case, the unbounded flow case $(\beta=0)$ has also been considered. In this case, a symmetry boundary condition was assigned along the $y=H$ line, and from numerical experiments it was concluded that $H=60 a$ was sufficient to guarantee results independent of the size adopted for the flow domain. The mesh is similar to that of Fig. 24(a), with only 4 mesh defining blocks and non-uniform mesh spacing; the number of cells was now 6400 and the minimum spacing was $\delta_{x} /(2 a)=0.077$. The Newtonian flow case at a Reynolds number of 1 was solved firstly, yielding $C_{\mathrm{D}}=11.4$ which compares well with the value of 10.7 predicted by Huang and Feng and also with an empirical expression from the literature. With the Reynolds number held constant at a value of 1 , the
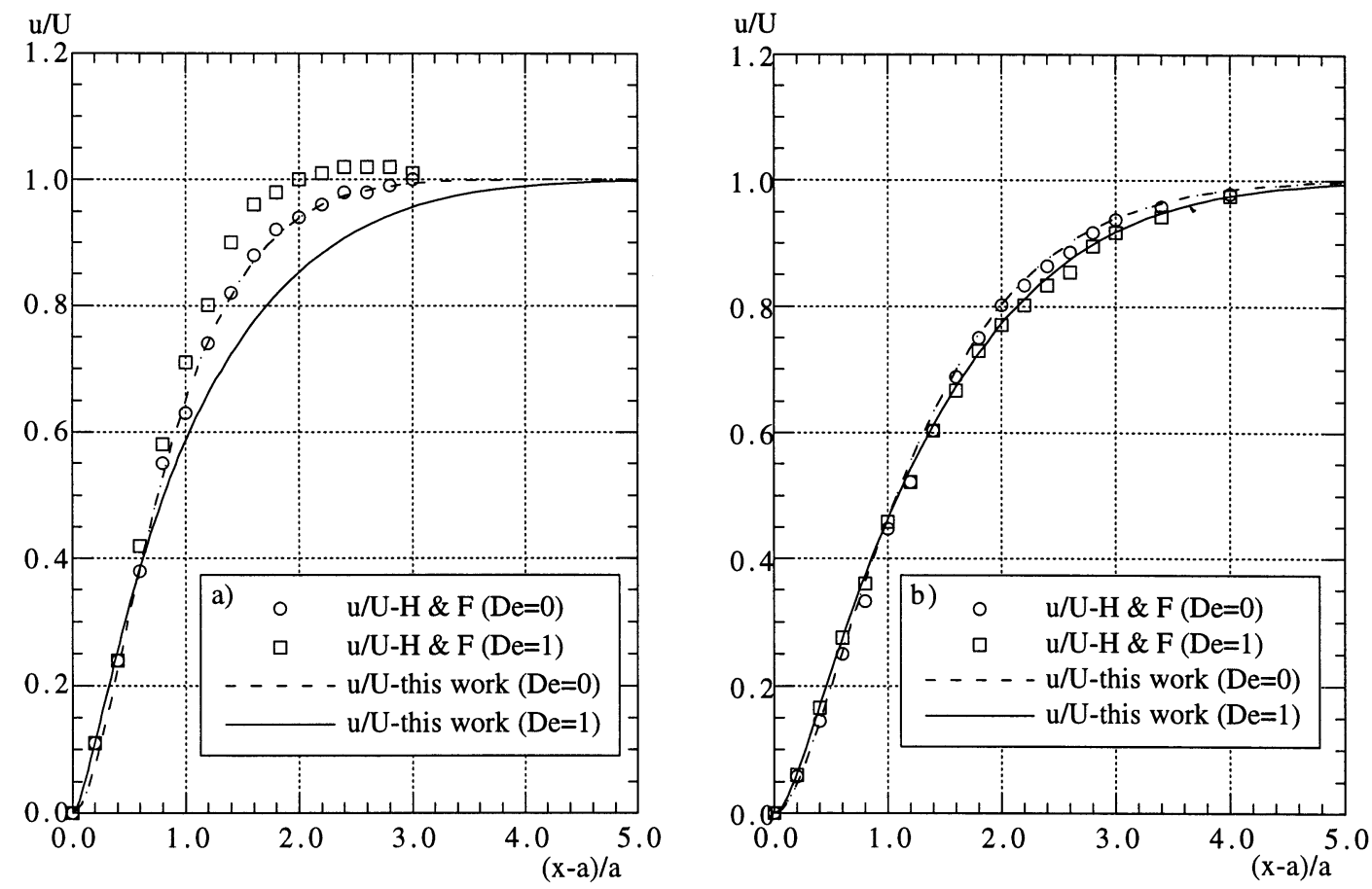

Fig. 26. Longitudinal velocity along the centerline in the wake of the bounded cylinder: (a) $\beta=0.5$; (b) $\beta=0.33$. 


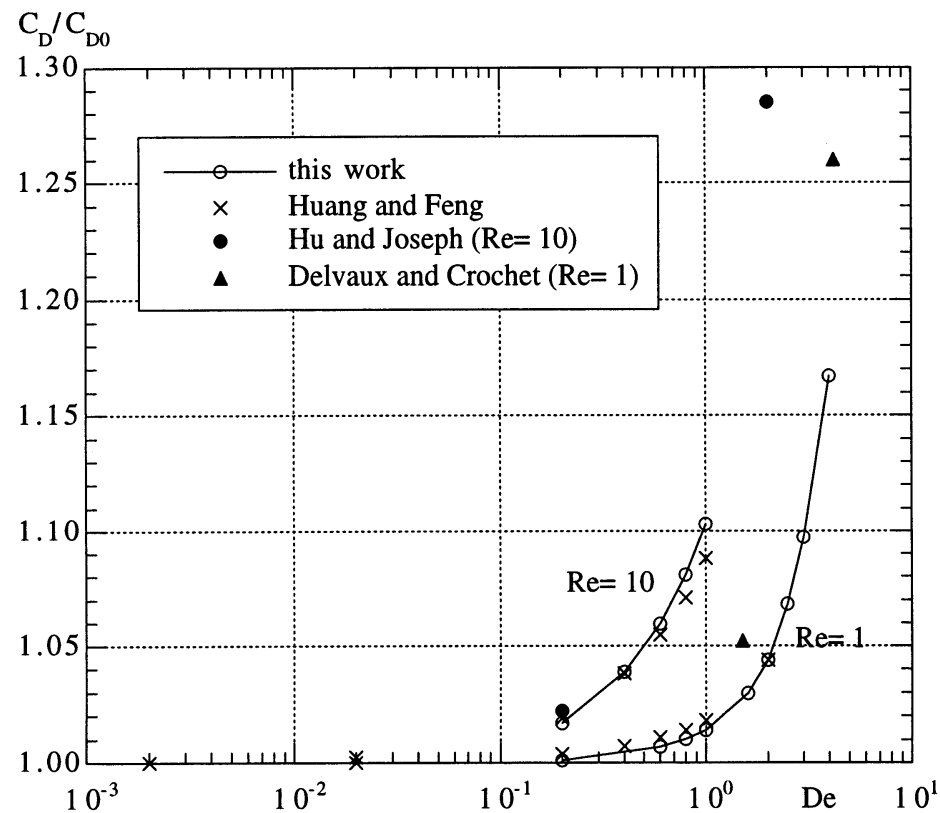

Fig. 27. Ratio of the viscoelastic to the Newtonian drag coefficient as a function of the Deborah and Reynolds numbers.

relaxation time was successively increased up to a Deborah number of 4 (a value higher than that in Huang and Feng, however, the maximum Deborah number for iterative convergence may depend on the mesh fineness) and the resulting drag coefficients, relative to their Newtonian counterpart $\left(C_{\mathrm{D}_{\mathrm{o}}}\right)$, are plotted in Fig. 27 together with those of Huang and Feng. A higher Reynolds number case $(\mathrm{Re}=10)$ has been considered and the results are also given in Fig. 27. As expected, the agreement now is quite good, both results showing a progressive increase of the drag coefficient with elasticity, and the discrepancy between the upper convected Maxwell and the Oldroyd-B fluids seen earlier for the larger blockage ratio is absent in this unbounded case. This is in agreement with Delvaux and Crochet's [29] findings.

\section{Conclusions}

A new finite-volume methodology for the computation of the flow of viscoelastic fluids has been developed and is presented here in some detail. The methodology is general in the sense that both 2D and 3D simulations can be performed and the finite-volume mesh may be non-orthogonal and semi-structured to conform to any arbitrary flow-boundary geometry.

The interpolation techniques used for all terms in the continuity, momentum and constitutive equations are second order accurate with some degree of upwind for the first derivative terms. The problem of the pressure-velocity-stress coupling was addressed and a new interpolation technique, inspired on that developed by Rhie and Chow [1] for Newtonian flows have been designed for the stress terms appearing in the momentum equation. 
In order to assess the performance and accuracy of the method, calculations have been carried out for a channel entry flow of an upper convected Maxwell model at a Reynolds number of 20 and Deborah numbers between 0.1 and 1.0, and the results are in good agreement with those given by [2]. Very fine computational meshes have been used, compared with those generally found in viscoelastic computations; the minimum non-dimensional mesh spacing near the boundary discontinuity to the slip-stick problem was 0.005 (compare with 0.02 in Eggleton et al., who already used a very fine mesh).

Grid refinement studies have shown that the profiles of the calculated quantities are smooth (in contrast to [2]) and that grid independent values can be obtained except in the near vicinity of the wall discontinuity, a singular point where stress gradients tend to infinity, as implied theoretically. The new approach developed to obtain stress values at cell faces is shown to be effective in that it eliminates oscillations in the calculated profiles for the various quantities. Furthermore, iterative convergence was faster than that obtained with the simpler linear interpolation scheme. Convergence was attained for Deborah and Reynolds numbers identical or higher to those reported in the literature for the same flow problem, but in which lower accuracy methods have been used, namely for Deborah numbers in excess of 1.0 and 0.5 for a Reynolds number of 20 and 100, respectively. It should be noted that the purpose here has not been to investigate the range of applicability in terms of Deborah numbers of the new method.

Calculations for the same problem have been carried out using a $30^{\circ}$ skewed non-orthogonal grid at $\mathrm{De}=0.1, \mathrm{Re}=20$ and with the LUDS interpolation scheme. The results were virtually indistinguishable from those obtained with the orthogonal mesh.

The mean flow pattern for a slightly viscoelastic fluid $(\mathrm{De}=0.1)$ shows little difference to that of a Newtonian fluid and the viscoelastic fluids are seen to react to the approaching wall later than the Newtonian fluid in terms of the stress field. However, as the wall is approached, the viscoelastic fluid develops higher stresses and gradients of stress are more effective in decelerating the fluid in the near-wall region. Downstream of the slip-stick, the longitudinal normal stress of the viscoelastic fluids is tensile and very intense, due to the release of the stored elastic energy, whereas it is compressive and one order of magnitude smaller for Newtonian fluids. The fluid deceleration effect in the near-wall region is more pronounced with higher viscoelastic fluids, thus reducing the transverse transfer of momentum and delaying flow redevelopment.

The flow around a circular cylinder in a channel and in an infinite medium have also been considered in order to assess the performance of the methodology for non-orthogonal geometries. For the unbounded flow case and the confined case with area blockage of 0.33 , the results agree with those in the literature; for the higher blockage case $(\beta=0.5)$, discrepancies are found in the wake velocities which may be attributable to the different fluid rheologies and its influence upon the response of viscoelastic fluids to intense local shear and elongational flows due to the channel wall proximity.

Future developments include the adoption of more realistic viscoelastic models, application to various problems in which the range of applicability in terms of De and Re numbers should be assessed, and improvements in the performance of the numerical method, especially when operating with second- and higher-order accurate differencing schemes. 


\section{Acknowledgements}

The authors gratefully acknowledge the financial support of Junta Nacional de Investigação Científica e Tecnológica (JNICT, Portugal) through project PBIC/C/QUI1980/95, and the lab facilities provided by Instituto de Engenharia Mecânica e Gestão Industrial (INEGI). The authors are listed alphabetically.

\section{References}

[1] C.M. Rhie, W.L. Chow, A numerical study of the turbulent flow past an isolated airfoil with trailing edge separation, ALAA 82 (1982) 998.

[2] C.D. Eggleton, T.H. Pulliam, J.H. Ferziger, Numerical simulation of viscoelastic flow using flux differene splitting at moderate Reynolds numbers, J. Non-Newtonian Fluid Mech. 64 (1996) 269.

[3] X. Huang, N. Phan-Thien, R.I. Tanner, Viscoelastic flow between eccentric rotating cylinders: unstructured control volume method, J. Non-Newtonian Fluid Mech. 64 (1996) 71.

[4] S.V. Patankar, Numerical Heat Transfer and Fluid Flow, McGraw-Hill, New York, 1980.

[5] J.H. Ferziger, M. Peric, Computational Methods for Fluid Dynamics. Springer, Berlin, 1996.

[6] H.H. Hu, D.D. Joseph, Numerical simulation of viscoelastic flow past a cylinder, J. Non-Newtonian Fluid Mech. 37 (1990) 347

[7] J.Y. Yoo, Y. Na, A numerical study of the planar contraction flow of viscoelastic fluids using the SIMPLER algorithm, J. Non-Newtonian Fluid Mech. 39 (1991) 89.

[8] G.D. Raithby, A critical evaluation of upstream differencing applied to problems involving fluid flow, Comput. Meth. Appl. Mech. Eng. 9 (1976) 75.

[9] B. Gervang, P.S. Larsen, Secondary flows in straight ducts of rectangular cross section, J. Non-Newtonian Fluid Mech. 39 (1991) 217.

[10] G.P. Sasmal, A finite volume approach for calculation of viscoelastic flow through an abrupt axisymmetric contraction, J. Non-Newtonian Fluid Mech. 56 (1995) 15.

[11] S.-C. Xue, N. Phan-Thien, R.I. Tanner, Numerical study of secondary flows of viscoelastic fluid in straight pipes by an implicit finite volume method, J. Non-Newtonian Fluid Mech. 59 (1995) 192.

[12] M. Vinokur, An analysis of finite-difference and finite-volume formulations of conservation laws, J. Comput. Phys. 81 (1989) 1.

[13] G.A. Pinto, Development of a Numerical Simulation Program for the Calculation of Laminar Flows with Non-Linear Elastic Fluids (in portuguese). MSc. Thesis, University of Porto, Portugal, 1996.

[14] P.K. Khosla, S.G. Rubin, A diagonally dominant second-order accurate implicit scheme, Comput. Fluids 2 (1971) 207.

[15] R.I. Issa, P.J. Oliveira, Numerical predictions of phase separation in two-phase flow through T-junctions, Comput. Fluids 23 (1994) 347.

[16] S.V. Patankar, D.B. Spalding, A calculation procedure for heat, mass and momentum transfer in three-dimensional parabolic flows, Int. J. Heat Mass Transfer 15 (1972) 1787.

[17] J.P. Van Doormal, G.D. Raithby, Enhancements of the SIMPLE method for predicting incompressible fluid flows, Num. Heat Transfer 7 (1984) 147.

[18] J.A. Meijerink, H.A. Van Der Vorst, An iterative solution method for linear systems of which the coefficient matrix is a symmetric M-matrix, Math. Comput. 31 (1977) 148.

[19] P.J. Oliveira, Boundary treatment of stress equation. Internal report. Departmento de Electromecânica, Universidade da Beira Interior, Rua Marquês D’Ávila e Bolama, 6200 Covilhã, Portugal, 1996.

[20] P.Y. Huang, J. Feng, Wall effects on the flow of viscoelastic fluids around a circular cylinder, J. Non-Newtonian Fluid Mech. 60 (1995) 179.

[21] R.C. King, M.R. Apelian, R.C. Armstrong, R.A. Brown, Numerically stable finite element techniques for viscoelastic calculations in smooth and singular geometries, J. Non-Newtonian Fluid Mech. 29 (1988) 147. 
[22] M.J. Crochet, Numerical simulation of viscoelastic flow, Lecture Series 1994-03, Von Karman Institute of Fluid Dynamics, 1994.

[23] R.A. Brown, G.H. McKinley, Report on the eight international workshop on numerical methods in viscoelastic flows, J. Non-Newtonian Fluid Mech. 52 (1994) 407.

[24] J. Sun, N. Phan-Thien, R.I. Tanner, An adaptive viscoelastic stress splitting scheme and its applications: AVSS/SI and AVSS/SUPG, J. Non-Newtonian Fluid Mech. 65 (1996) 75.

[25] M.B. Bush, The stagnation flow behind a sphere, J. Non-Newtonian Fluid Mech. 49 (1993) 103.

[26] O. Manero, B. Mena, On the slow flow of viscoelastic liquids past a circular cylinder, J. Non-Newtonian Fluid Mech. 9 (1981) 379.

[27] F.P.T. Baaijens, Application of low-order discontinuous Galerkin methods to the analysis of viscoelastic flows, J. Non-Newtonian Fluid Mech. 52 (1994) 37.

[28] R.B. Bird, R.C. Armstrong, O. Hassager, Dynamics of Polymeric Liquids, 2nd ed., vol. 1: Fluid Mechanics, Wiley, New York, 1987.

[29] V. Delvaux, M.J. Crochet, Numerical predictions of anomalous transport properties in viscoelastic flow, J. Non-Newtonian Fluid Mech. 37 (1990) 297. 